\title{
Une lecture institutionnelle des différences de qualité du travail et de l'emploi entre la France et les États-Unis
}

An institutional analysis of job quality discrepancies in France and in the United States

\section{Charlotte Levionnois}

\section{OpenEdition \\ Journals}

Édition électronique

URL : http://journals.openedition.org/travailemploi/6617

DOI : 10.4000/travailemploi.6617

ISSN : 1775-416X

Éditeur

DARES - Ministère du Travail

Édition imprimée

Date de publication : 1 avril 2015

Pagination : 21-39

ISSN : 0224-4365

Référence électronique

Charlotte Levionnois, « Une lecture institutionnelle des différences de qualité du travail et de l'emploi entre la France et les États-Unis », Travail et Emploi [En ligne], 142 | avril-juin 2015, mis en ligne le 01 avril 2017, consulté le 20 avril 2019. URL : http://journals.openedition.org/travailemploi/6617 ; DOI $10.4000 /$ travailemploi.6617 


\title{
Une lecture institutionnelle des différences de qualité du travail et de l'emploi entre la France et les États-Unis
}

\author{
Charlotte Levionnois ${ }^{*}$ )
}

Cet article propose une comparaison franco-américaine de la qualité de l'emploi des enfants d'immigrés. En utilisant l'enquête Emploi en continu et l'enquête sur la population américaine (Current Population Survey) entre 2008 et 2012, nous analysons trois dimensions de la qualité du travail et de l'emploi : le salaire, la sécurité de l'emploi et le temps de travail. L'analyse met en évidence une qualité de l'emploi des enfants d'immigrés en moyenne inférieure à celle des natifs dans les deux pays, et qui est par ailleurs très hétérogène au sein du groupe des descendants d'immigrés. Nous montrons que les écarts observés sont plus souvent imputables à l'origine géographique en France qu'aux États-Unis. Nos résultats laissent penser que si les institutions françaises assurent un niveau de qualité de l'emploi globalement supérieur, elles s'accompagnent d'inégalités plus marquées entre natifs et descendants d'immigrés et impliquent une plus forte barrière à l'entrée dans l'emploi.

De nombreuses études comparatives montrent que la qualité de l'emploi, concept multidimensionnel abordé ici dans une perspective de sécurité socio-économique, est hétérogène au sein des pays de l'Organisation de coopération et de développement économiques (OCDE, 2013a; SchmitT, 2012). Des liens entre qualité de l'emploi et institutions du marché du travail ont notamment été mis en évidence : la dépense publique par chômeur (qui correspond aux politiques de l'emploi) ou l'existence d'un salaire minimum relativement élevé contribuent par exemple à réduire les risques de dégradation de la qualité de l'emploi des individus (Gautié, Schmitt, 2010). Par ailleurs, les travaux sur ce sujet mettent en lumière une hétérogénéité entre individus d'un même pays. Certaines caractéristiques individuelles sont défavorables en matière de qualité de l'emploi : les femmes et les peu qualifiés ont généralement une qualité de l'emploi inférieure aux autres individus dans l'ensemble des économies développées (OCDE, 2013a). Cette hétérogénéité est d'autant plus marquée dans les pays libéraux, où les institutions $\mathrm{du}$ marché du travail sont théoriquement «peu inclusives ${ }^{(1)} \gg$.

(*) Centre d'économie de la Sorbonne; Centre d'études de l'emploi; charlotte.levionnois@univ-paris1.fr

(1) «Les systèmes dits "inclusifs" utilisent la négociation collective, le droit national, et même les normes sociales - des mécanismes volontaires qui régissent le comportement des entreprises - pour étendre le pouvoir de négociation individuelle et collective des travailleurs à hauts salaires à ceux faiblement rémunérés, ce qui permet souvent à ces travailleurs à bas salaires de franchir les seuils de bas salaires. » (APPELBAUM, Sснмiтt, 2009, p. 1909; nous traduisons.)
La qualité de l'emploi des populations immigrées et issues de l'immigration a fait l'objet de peu d'études en soi. Pourtant, même si les positions sur le marché du travail des descendants d'immigrés sont meilleures que celles de leurs parents, des inégalités entre populations descendant de l'immigration et natives peuvent être relevées dans la littérature. Les inégalités apparaissent dès la spécialisation par le diplôme et le niveau d'étude (Alba, Holdaway, 2013). Elles se retrouvent ensuite sur le marché du travail : certaines études ont par exemple mis en lumière les discriminations à l'embauche envers les immigrés et leurs descendants par rapport aux natifs. Ces inégalités dans l'accès à l'emploi peuvent être favorisées par une réglementation peu contraignante du travail, comme dans les pays libéraux, notamment aux États-Unis (OCDE, 2013b; Sснмiтt, 2012). Elles peuvent également renvoyer à d'autres politiques publiques, comme celles portant plus spécifiquement sur l'intégration des populations issues de l'immigration.

L'analyse comparative de la qualité de l'emploi des descendants d'immigrés proposée dans cet article s'intéresse à la France et aux ÉtatsUnis. Ces deux pays présentent des similarités démographiques puisque la proportion d'enfants d'immigrés est en 2012 de 9,8\% dans la population française en âge de travailler et de $8,4 \%$ dans la population américaine correspondante ( $c f$. infra pour une présentation des données utilisées dans l'article). La France et les États-Unis ont à l'inverse deux ensembles très différents d'institutions du marché du travail, qui sont protectrices mais 
duales( ${ }^{(2)}$ en France et nettement moins protectrices aux États-Unis (GALlie, 2007), et de deux modèles d'intégration à bien des égards opposés : l'un étant «républicain » (en France) quand l'autre est «multiculturaliste» (aux États-Unis; WeIL, 2003). Les effets attendus de la combinaison des institutions du marché du travail et des modèles d'intégration sur les inégalités en termes de qualité de l'emploi des descendants d'immigrés sont complexes. Comment les effets protecteurs des institutions du marché du travail françaises influencent-ils les différences de qualité de l'emploi entre sous-populations ? Est-ce que les politiques américaines d'intégration plus volontaristes permettent d'améliorer les écarts selon l'origine des parents?

Dans cet article, nous tentons de préciser empiriquement les différences de qualité de l'emploi existant entre descendants d'immigrés et natifs. Nous testons ensuite l'existence d'inégalités de qualité reposant sur des critères d'origine et proposons des hypothèses d'interprétation de nature institutionnelle. Nous ne prétendons pas à une analyse causale des liens entre institutions et qualité de l'emploi des descendants d'immigrés, mais soumettons à la discussion plusieurs hypothèses expliquant les différences entre les deux pays.

Pour ce faire, la première partie revient sur le concept de qualité de l'emploi et ses composantes, puis présente un état de la littérature consacrée à l'intégration sur le marché du travail des enfants d'immigrés. Dans une deuxième partie, nous détaillons nos données et la stratégie empirique adoptée. Enfin, la troisième partie propose une analyse économétrique des effets de l'origine sur l'accès à l'emploi et la qualité de l'emploi en France et aux États-Unis, suivie d'une discussion des résultats à partir d'un cadre d'analyse institutionnel comparatif.

\section{La qualité de l'emploi en France et aux États-Unis}

\section{La qualité de l'emploi : une notion multidimensionnelle}

La littérature économique et les organisations internationales s'accordent pour considérer la qualité de l'emploi comme un concept multidimensionnel. Nous mettons ici l'accent sur trois dimensions essentielles liées à la sécurité socioéconomique retenues dans les travaux académiques

(2) Le dualisme du marché du travail peut par exemple être défini par une "coexistence d'un secteur primaire caractérisé par de hauts salaires et la sécurité de l'emploi, et un secteur secondaire dans lequel les salaires sont faibles et le risque de chômage important. »(CAHUC, ZAJDELA, 1991, p. 469.) et les définitions proposées par les organisations internationales (UNECE, 2010; ILO, 2012) : le salaire, la sécurité de l'emploi et le temps de travail.

Le salaire constitue la composante la plus souvent intégrée à l'analyse de la qualité de l'emploi dans la mesure où il peut permettre aux travailleurs d'acquérir un niveau de vie décent. De nombreux travaux se sont concentrés sur le lowwage work (Appelbaum, SсHмitt, 2009; Caroli, Gautié, 2009; Gautié, Schmitt, 2010), en particulier aux États-Unis où les inégalités de revenu et de salaire ont explosé au XXe siècle (PiketTy, SAez, 2003). Plus de $20 \%$ des travailleurs y gagnent un salaire inférieur au seuil de pauvreté (OSTERMAN, Shulman, 2011; article de Paul Osterman dans ce numéro). Cette littérature souligne qu'en France, la part de travailleurs pauvres est plus faible en raison d'institutions du marché du travail plus «inclusives» et notamment de l'existence et du niveau du salaire minimum (Appelbaum, Schmitt, 2009).

Un deuxième pan de la littérature établit une distinction entre good jobs et bad jobs, qui est principalement déterminée par le niveau de sécurité de l'emploi. En plus d'envisager les niveaux de salaire, les analyses prennent ainsi en compte le type de contrat de travail, en distinguant les emplois temporaires des emplois à durée indéterminée (CDI). En France, cette distinction est particulièrement pertinente étant donné la différence de sécurité de l'emploi existant entre emplois à durée indéterminée (CDI ou titulaires de la fonction publique) et déterminée (CDD, contrats saisonniers, contrats d'intérim ou de travail temporaire, contrats d'apprentissage, contrats en alternance). Aux États-Unis, l'écart de sécurité entre les différents emplois ne se joue pas tant sur le contrat de travail que sur l'accès à l'assurance-santé. Alors qu'en France l'assurance-santé est universelle, cette dernière varie en fonction du contrat de travail aux États-Unis. C'est donc l'accès à ces formes de prestations sociales associées au contrat de travail, les benefits ${ }^{(3)}$, qui constitue, pour les Américains, un critère déterminant de la sécurité de leur emploi (KALLEBERG et $a l ., 2000$; Schmitt, Jones, 2012).

Les deux premières dimensions de la qualité de l'emploi (salaire et sécurité de l'emploi) sont intimement liées à une troisième, le temps de travail. En effet, malgré leur diversité (UlRICH, ZILBERMAN, 2007), les emplois à temps partiel assurent généralement un salaire assez faible en raison du faible nombre d'heures travaillées et offrent moins de sécurité - que ce soit aux salariés français, qui

(3) Les benefits sont des prestations annexes versées par l'employeur telles qu'une contribution à une assurance-maladie ou une pension complémentaire retraite. 
sont moins souvent en contrat à durée indéterminée, ou aux travailleurs américains en termes $\mathrm{d}$ 'accès aux benefits. Les emplois à temps partiel sont de plus en plus utilisés par les entreprises dans une logique de flexibilité (ILO, IILS, 2008). Ils peuvent constituer des emplois précaires, que les travailleurs choisissent parfois pour des raisons personnelles mais qu'ils subissent lorsque leurs opportunités d'emploi à temps plein sont inexistantes.

\section{Les apports de la littérature sur les inégalités selon l'origine}

Dans les deux pays, l'intégration des descendants d'immigrés sur le marché du travail a fait l'objet de nombreuses recherches : de leur employabilité à certaines caractéristiques spécifiques de leurs emplois, l'objet principal d'investigation restant toutefois leur salaire. Ces études, qui comparent la situation des natifs à celle des descendants d'immigrés, ont mis en évidence l'existence d'inégalités liées à l'origine, notamment dans l'accès à l'emploi ou sur le salaire.

Cette hétérogénéité des situations en fonction de l'origine se retrouve dans les deux pays; d'abord aux États-Unis, où les premiers travaux sur l'ethnicité ont vu le jour, avec l'ouvrage de Nathan GLAzER et Daniel P. Moynihan (1963). En France, Roxane Silberman et Irène FourniER (2006) ont mis en évidence une "pénalité ethnique ${ }^{(4)}$ » pour les descendants d'immigrés, dont les plus touchés sont les Maghrébins, les Turcs et les Africains originaires de la zone subsaharienne. Les études sur le degré de sécurité de l'emploi indiquent par ailleurs une plus forte vulnérabilité à l'emploi précaire de certains descendants d'immigrés : plus de $25 \%$ de ceux ayant une origine maghrébine ou subsaharienne occupent des emplois précaires (Meurs, et al., 2006). À l'inverse, certaines populations issues de l'immigration (première et seconde génération) sont relativement bien intégrées professionnellement, par exemple celles venant du Portugal (Meurs et al., 2006).

La littérature souligne l'importance de la structure des emplois pour expliquer ces inégalités entre natifs et descendants d'immigrés mais aussi entre groupes d'origine différente (Butcher, DiNardo, 1998; Aeberhardt, Pouget, 2007). Romain AEBERHARDT et ses coauteurs (2010) chiffrent l'écart salarial entre les descendants d'immigrés maghrébins et les natifs à $13 \%$, l'hypothèse d'une ségrégation professionnelle plutôt qu'une discrimination salariale étant par ailleurs avancée comme principale explication (Aeberhardt, Pouget, 2007). C'est aussi ce que suggèrent Dominique Meurs et ses coauteurs (2006) à l'aide de l'enquête Étude de l'histoire familiale, mettant en évidence une ségrégation professionnelle de certains descendants d'immigrés dans les emplois peu rémunérés. Dans certains secteurs d'activité, on compte de nombreux descendants d'immigrés d'une même origine, ce qui induit une spécialisation de fait : en France, tel est le cas des immigrés portugais et de leurs descendants dans la construction et les secteurs de petite industrie ou des jeunes hommes d'origine maghrébine dans le secteur des services (LaInÉ, OKвA, 2005). Ces orientations peuvent à terme conduire les travailleurs à occuper des emplois dans des secteurs à la qualité de l'emploi très variable.

Dans l'ensemble, la littérature suggère ainsi une forte hétérogénéité entre origines, qu'il conviendra de tester dans notre analyse empirique non seulement s'agissant de l'accès à l'emploi mais aussi des caractéristiques de ce dernier.

\section{Comparaison franco-américaine des composantes de la qualité de l'emploi}

\section{Données et méthode}

\section{La mesure de la qualité de l'emploi en pratique}

Notre comparaison empirique repose sur l'exploitation de deux bases de données. D'une part, nous utilisons l'enquête Emploi en continu (EEC) de l'Institut national de la statistique et des études économiques (Insee) pour la France de 2008 à 2012, ce qui nous permet de disposer d'un échantillon représentatif, adapté aux décompositions selon l'origine des parents. D'autre part, et de façon similaire, nous utilisons le supplément mensuel de mars de la Current population survey (CPS), intitulé Annual Social and Economic (Asec) de 2008 à 2012 pour les États-Unis. L'enquête CPS est conduite mensuellement par le Census Bureau (CB) et fournit plus précisément, dans le supplément de mars, des informations détaillées sur les caractéristiques des individus et leur situation sur le marché du travail. Ces deux bases de données permettent l'identification précise des descendants d'immigrés (voir encadré). 


\section{Encadré}

Les descendants d'immigrés dans l'enquête Emploi en continu et la Current Population Survey

Un descendant d'immigré est, dans le cas français, une personne résidant en France et née en France d'au moins un parent immigré, c'est-à-dire né étranger à l'étranger. De la même manière, aux États-Unis, un descendant d'immigré réside dans le pays, y est né, et a au moins un de ses parents qui est immigré.

En France, contrairement à la plupart des pays et à la pratique internationale qui se base sur le pays de naissance indépendamment de la nationalité à la naissance, une personne présente en France, née à l'étranger mais de nationalité française à la naissance n'est pas considérée comme immigrée ${ }^{(1)}$.

Dans cet article, le pays de naissance du père ou de la mère immigré(e) détermine l'origine géographique. Dans les cas où les deux parents sont immigrés, c'est le pays du père qui est choisi (MıNNI, OKBA, 2014). Nous nous concentrons ici sur les situations des descendants d'immigrés par rapport à celles des natifs, c'est-à-dire des personnes nées en France dont aucun des deux parents n'est immigré.

(1) «Il existe un cas particulier quand les parents sont nés en Algérie avant l'indépendance (1962) mais ont décidé de rester en Algérie et de garder la nationalité algérienne à ce moment. En cas de questions sur leurs origines, ces parents doivent se déclarer algériens de naissance nés en Algérie (la question du pays de naissance concerne toujours les pays dans leurs frontières actuelles), soit étrangers nés à l'étranger. S'ils ont migré en France par la suite et ont donné naissance à un enfant, ce dernier naît français selon le principe du double droit du sol (les parents et l'enfant sont nés en France, l'Algérie étant un département français avant 1962).» (BREEM, 2010, p. 3.)

Notre étude de la qualité de l'emploi repose sur l'analyse des trois dimensions présentées dans la section précédente (salaire, sécurité de l'emploi, temps de travail) ${ }^{(5)}$. Travailler sur des dimensions désagrégées permet de conserver au maximum l'information disponible (GUERGOAT-LARIVIĖRE, Marchand, 2012; Osterman, 2013). Ces trois dimensions correspondent aux caractéristiques de l'emploi mais ne couvrent pas celles intrinsèques du travail, comme les conditions de travail, notamment en raison de la disponibilité des données. Ne sont par ailleurs considérés que les emplois du secteur privé, les fonctionnaires étant exclus du champ d'étude.

(5) L'analyse est réalisée pour l'emploi principal des individus. Les emplois secondaires ne sont pas étudiés.
Les mesures de ces dimensions peuvent différer entre la France et les États-Unis en raison des spécificités institutionnelles nationales. Par exemple, pour la France, la dimension sécurité de l'emploi s'appuie sur la nature du contrat de travail, avec deux modalités : l'une pour laquelle l'emploi a une sécurité élevée (les CDI) et l'autre, à faible sécurité de l'emploi, qui comprend les CDD, les contrats saisonniers, les contrats en apprentissage et en alternance et les contrats de travail temporaire et d'intérim. Aux États-Unis, la dimension relative à la sécurité de l'emploi repose sur la mise à disposition de la part des employeurs d'une assurance-santé. La dimension temps de travail est construite de façon proche dans les deux pays grâce à une variable à deux modalités : l'une qui comprend le temps complet( ${ }^{(6)}$ et le temps partiel choisi et l'autre, le temps partiel subi. Pour le salaire, nous utilisons la même variable de salaire net mensuel en France et aux États-Unis.

\section{La mise en évidence du rôle de l'origine sur la qualité de l'emploi et la prise en compte de la sélection à l'entrée}

Notre stratégie empirique repose sur un modèle d'Heckman (Heckman, 1998) qui permet de tenir compte du degré différent de sélection ${ }^{(7)}$ des natifs et des descendants d'immigrés pour accéder à un emploi. Dans une première étape, une régression de type probit binomial est réalisée pour estimer la probabilité d'être en emploi avec l'introduction d'une variable de sélection. À la suite de nombreux travaux consacrés à l'estimation de modèles de participation au marché du travail, nous utilisons les caractéristiques du ménage - situation matrimoniale et nombre d'enfants - comme variables d'exclusion (Mroz, 1987; Hyslop, 1999 et Buchinsky et al., 2010). Nous distinguons d'abord dans notre modèle les descendants d'immigrés des natifs puis, pour prendre en compte l'hétérogénéité entre origines, l'analyse est désagrégée par origine des parents. Dans une seconde étape, nous estimons trois régressions de qualité de l'emploi : une régression linéaire pour le salaire et deux probits binomiaux ${ }^{(8)}$ pour la sécurité de l'emploi et le temps de travail.

Nous introduisons de nombreux contrôles dans les analyses économétriques. D'abord, nous contrôlons par le genre, les femmes étant largement pénalisées en termes de qualité de l'emploi en France mais aussi aux États-Unis (Davoine, ERHEL, 2007; KalleberG et al., 2000). Nous contrôlons par ailleurs par l'âge,

(6) Aux États-Unis, le temps plein est défini comme un emploi de 35 heures par semaine (temps de travail plein fédéral) même si, en pratique, cette durée est parfois considérée comme partielle (voir le glossaire en ligne du Bureau of Labor Statistics : http://www.bls.gov/bls/glossary.htm\#F; page consultée le 24 août 2015).

(7) On postule la normalité jointe des termes d'erreur.

(8) Dans le cas où la variable expliquée est qualitative et binaire, on parle de probits bivariés avec sélection. 
ce qui permet à la fois de tenir compte de la structure d'âge différente des populations et de capter les difficultés rencontrées par les jeunes notamment (LE Rhun, Minni, 2012). Supposant que l'âge n'a pas un impact linéaire - les seniors se trouvent également en difficulté (ibid.) - nous contrôlons par l'âge sous une forme quadratique. Nous prenons aussi en compte le niveau de diplôme afin de considérer les effets de structure, puisque les individus les plus diplômés ont en moyenne une meilleure qualité de l'emploi (Le Rhun, Pollet, 2011) ${ }^{(9)}$. Une variable sur le fait d'avoir au moins un parent non immigré (i.e. français ou américain) est construite afin de contrôler du potentiel différentiel d'intégration selon que l'individu ait bénéficié ou non de la culture française ou américaine par au moins l'un de ses parents (AlgAN et al., 2012). En raison des disparités territoriales dans les deux pays (RATHELOt, 2010; BoRJAs, 1995), une variable de résidence est introduite: elle tient compte du fait de vivre dans une aire métropolitaine aux États-Unis et, en France, de celui d'habiter en région parisienne et en zone urbaine sensible (ZUS). Enfin une dernière variable de contrôle portant sur le secteur d'activité de l'emploi a été ajoutée pour contrôler des disparités sectorielles en termes de qualité de l'emploi.

\section{Qui sont les descendants d'immigrés ?}

\section{Portrait des descendants d'immigrés}

Dans le cadre de cette comparaison institutionnelle, nous nous concentrons sur les descendants d'immigrés, qui représentent une frange importante de la population des deux pays et présentent des caractéristiques sociodémographiques spécifiques.

\section{Graphique 1 : Répartition des descendants d'immigrés selon l'origine de leurs parents en France}

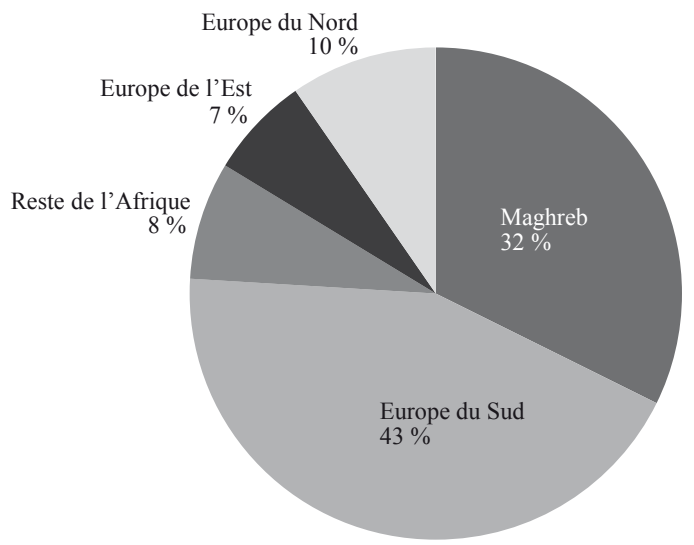

Champ: Descendants d'immigrés, de 20 à 60 ans, en France métropolitaine.

Source : Enquête Emploi en continu 2012, Insee.

(9) L'hypothèse d'un effet de composition a aussi été testée au moyen de deux contrôles supplémentaires (part des jeunes et part des non-diplômés) dans des régressions non incluses dans l'article pour des contraintes de place.

\section{Graphique 2 : Répartition des descendants d'immigrés selon l'origine de leurs parents aux États-Unis}

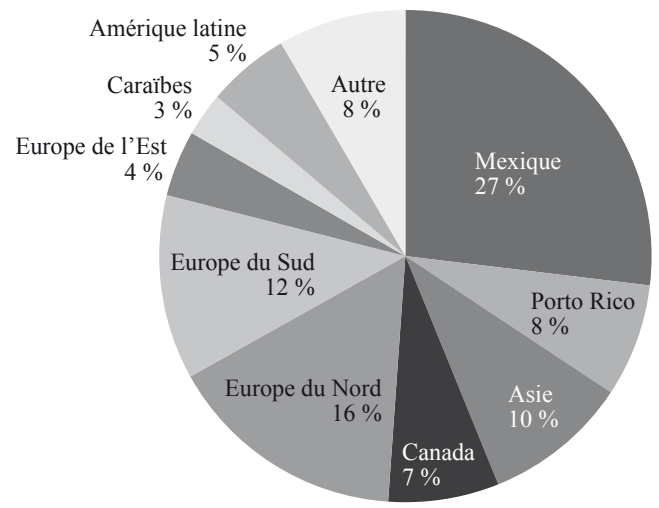

Champ : Descendants d'immigrés, de 20 à 60 ans, aux États-Unis. Source : Current Population Survey 2012, BLS.

La France et les États-Unis ont connu de fortes vagues migratoires entre le $\mathrm{XIX}^{\mathrm{e}}$ et le début du $\mathrm{xx}^{\mathrm{e}}$ siècle puis, de nouveau et surtout, dans les années 1960 (GREEN, 1991). Les descendants des immigrés d'après-guerre sont aujourd'hui nombreux sur le marché du travail des deux pays (voir graphiques 1 et 2 pour une répartition des enfants d'immigrés par origine). Ils sont en moyenne plus jeunes que les natifs (les descendants d'immigrés sont dans les deux pays plus nombreux à avoir moins de 25 ans, et moins nombreux au-delà de 35 ans en France et de 45 ans aux États-Unis [voir tableaux 1 et 2]). Ils ont également un niveau d'éducation plus faible: les tableaux 1 et 2 montrent que, par rapport aux natifs, une part plus importante des descendants d'immigrés est non diplômée (i.e. le certificat d'études primaires ou moins en France, et sortie du système scolaire au niveau de l'équivalent de la quatrième, sans diplôme, aux États-Unis) et qu'une part moins importante est diplômée du supérieur dans les deux pays. Enfin, les descendants d'immigrés habitent plus fréquemment en zone urbaine aux États-Unis et en zone urbaine sensible et/ou région parisienne en France. 
Tableau 1 : Portrait statistique des enfants d'immigrés en France en 2012

\begin{tabular}{|c|c|c|c|c|c|c|c|}
\hline & \multirow{2}{*}{$\begin{array}{c}\text { Français nés } \\
\text { de parents } \\
\text { français }\end{array}$} & \multirow[b]{2}{*}{$\begin{array}{c}\text { Enfants } \\
\text { d'immigrés }\end{array}$} & \multicolumn{5}{|c|}{ Dont } \\
\hline & & & Maghreb & $\begin{array}{l}\text { Europe } \\
\text { du Sud }\end{array}$ & $\begin{array}{l}\text { Reste de } \\
\text { l'Afrique }\end{array}$ & $\begin{array}{c}\text { Europe de } \\
\text { l'Est }\end{array}$ & $\begin{array}{c}\text { Europe } \\
\text { du Nord }\end{array}$ \\
\hline \multicolumn{8}{|l|}{ Sexe } \\
\hline Femme & 52,2 & 53,9 & 54,1 & 52,6 & 55,1 & 54,2 & 52,7 \\
\hline Homme & 47,8 & 46,1 & 45,9 & 47,4 & 44,9 & 45,8 & 47,3 \\
\hline \multicolumn{8}{|l|}{ Âge } \\
\hline Moins de 25 ans & 12,6 & 17,1 & 19,5 & 11,0 & 40,5 & 4,9 & 14,1 \\
\hline De 26 à 35 ans & 24,1 & 28,3 & 37,0 & 22,7 & 36,4 & 14,5 & 20,0 \\
\hline De 36 à 45 ans & 26,1 & 24,4 & 27,8 & 29,6 & 11,0 & 18,5 & 19,4 \\
\hline De 46 à 60 ans & 37,3 & 30,3 & 15,7 & 36,7 & 12,1 & 62,2 & 46,6 \\
\hline \multicolumn{8}{|l|}{ Diplôme } \\
\hline Pas de diplôme & 14,1 & 19,3 & 23,8 & 17,2 & 15,7 & 17,8 & 13,3 \\
\hline BEPC ou Brevet des collèges & 6,9 & 9,0 & 9,0 & 7,9 & 8,9 & 10,9 & 7,3 \\
\hline$C A P-B E P^{*}$ & 25,1 & 23,8 & 21,5 & 28,3 & 9,6 & 29,3 & 21,7 \\
\hline Baccalauréat professionnel & 7,1 & 7,1 & 7,5 & 8,6 & 6,3 & 2,8 & 5,2 \\
\hline Baccalauréat technologique & 4,9 & 4,9 & 5,5 & 4,8 & 8,4 & 3,1 & 4,8 \\
\hline Baccalauréat général & 8,5 & 9,9 & 8,0 & 8,5 & 21,9 & 10,4 & 12,0 \\
\hline Formations de santé $(\mathrm{Bac}+2)$ & 2,8 & 1,7 & 1,5 & 2,1 & 1,1 & 0,8 & 2,3 \\
\hline$B T S-D U T^{* *}$ & 11,0 & 9,4 & 9,6 & 10,0 & 9,0 & 9,2 & 9,9 \\
\hline Université : Bac +2 & 1,3 & 1,4 & 1,6 & 1,5 & 0,5 & 3,3 & 0,8 \\
\hline Université : $B a c+3$ & 5,6 & 3,9 & 4,5 & 2,8 & 4,8 & 3,9 & 5,9 \\
\hline Université : Bac +4 & 3,3 & 2,6 & 2,2 & 2,7 & 1,5 & 1,7 & 2,2 \\
\hline École (Bac +3 et plus $)$ & 3,9 & 2,3 & 1,9 & 1,9 & 0,8 & 2,1 & 6,2 \\
\hline$B a c+5$ et plus & 5,6 & 4,8 & 3,4 & 3,7 & 11,5 & 4,7 & 8,4 \\
\hline \multicolumn{8}{|l|}{ Type de ménage } \\
\hline Homme célibataire sans enfant & 14,7 & 12,7 & 11,7 & 13,5 & 12,8 & 10,0 & 14,2 \\
\hline Femme célibataire sans enfant & 13,0 & 12,5 & 8,7 & 13,5 & 11,4 & 17,7 & 12,8 \\
\hline Homme célibataire avec enfants & 13,3 & 16,9 & 18,1 & 16,8 & 23,6 & 12,2 & 11,2 \\
\hline Femme célibataire avec enfants & 15,6 & 18,9 & 22,2 & 16,4 & 28,0 & 12,9 & 12,9 \\
\hline Homme en couple sans enfant & 5,5 & 4,0 & 2,6 & 4,7 & 2,0 & 6,1 & 5,9 \\
\hline Femme en couple sans enfant & 6,9 & 6,1 & 4,6 & 5,7 & 4,5 & 11,0 & 9,9 \\
\hline Homme en couple avec enfants & 14,3 & 12,6 & 13,5 & 12,4 & 6,6 & 17,6 & 16,0 \\
\hline Femme en couple avec enfants & 16,7 & 16,4 & 18,6 & 17,0 & 11,3 & 12,7 & 17,1 \\
\hline \multicolumn{8}{|l|}{ Lieu de résidence } \\
\hline Habite en province, hors d'une ZUS & 83,1 & 69,8 & 60,7 & 78,7 & 42,8 & 79,4 & 81,9 \\
\hline Habite en Île-de-France, hors d'une ZUS & 16,0 & 27,6 & 33,3 & 20,8 & 54,4 & 19,0 & 17,1 \\
\hline Habite en province, dans une ZUS & 0,8 & 1,7 & 3,4 & 0,5 & 1,7 & 1,1 & 1,0 \\
\hline Habite en Île-de-France, dans une ZUS & 0,2 & 1,0 & 2,7 & 0,0 & 1,1 & 0,5 & 0,0 \\
\hline \multicolumn{8}{|l|}{ Situation sur le marché du travail } \\
\hline Actif occupé & 77,0 & 66,6 & 56,2 & 76,4 & 60,7 & 71,1 & 70,1 \\
\hline Chômeur (à la recherche d'un emploi) & 7,5 & 11,2 & 16,4 & 8,5 & 12,5 & 8,7 & 7,1 \\
\hline Autre chômeur au sens du BIT $T^{* * *}$ & 0,4 & 0,5 & 0,3 & 0,3 & 0,6 & 0,0 & 0,8 \\
\hline Autre personne sans emploi & 15,1 & 21,7 & 27,1 & 14,8 & 26,3 & 20,3 & 22,0 \\
\hline Nombre d'observations & 29195 & 3376 & 1092 & 1473 & 261 & 225 & 325 \\
\hline
\end{tabular}

${ }^{*}$ : Certificat d'aptitude professionnelle - Brevet d'études professionnelles.

${ }^{* *}$ : Brevet de technicien supérieur - Diplôme universitaire de technologie.

${ }^{* * *}$ : Bureau international du travail.

Lecture : Parmi les Français dont les parents sont nés français, 5,64\% sont titulaires d'un diplôme de niveau Bac +5 et plus.

Champ : Individus de nationalité française, ayant entre 20 et 60 ans, vivant en logement ordinaire et qui ne sont ni étudiant, ni retraité, ni travailleur indépendant.

Source : Enquête Emploi en continu 2012, Insee. 
Tableau 2 : Portrait statistique des enfants d'immigrés aux États-Unis en 2012

\begin{tabular}{|c|c|c|c|c|c|c|c|c|c|c|c|c|}
\hline & \multirow{3}{*}{ 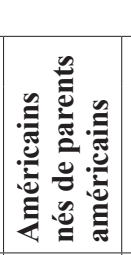 } & \multirow[b]{3}{*}{ 氠 } & \multirow{2}{*}{\multicolumn{10}{|c|}{ Dont (pays ou région d'origine des parents) }} \\
\hline & & & & & & & & & & & & \\
\hline & & & 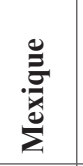 & 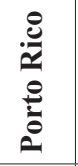 & $\frac{9}{4}$ & 胥 & 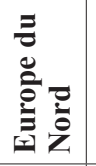 & 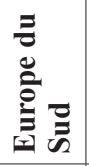 & 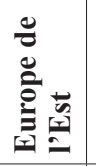 & 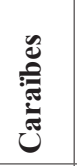 & 窇 & $\stackrel{\circlearrowright}{\Xi}$ \\
\hline \multicolumn{13}{|l|}{ Sexe } \\
\hline Femme & 50,1 & 51,5 & 51,8 & 56,8 & 49,6 & 49,2 & 49,2 & 52,6 & 43,0 & 59,5 & 54,7 & 52,7 \\
\hline Homme & 49,9 & 48,5 & 48,2 & 43,2 & 50,4 & 50,8 & 50,8 & 47,4 & 57,0 & 40,5 & 45,3 & 47,3 \\
\hline \multicolumn{13}{|l|}{ Âge } \\
\hline Moins de 25 ans & 3,4 & 6,6 & 12,4 & 2,5 & 5,5 & 3,1 & 2,5 & 2,2 & 0,3 & 4,0 & 15,9 & 8,1 \\
\hline De 26 à 35 ans & 20,5 & 29,3 & 38,1 & 23,5 & 46,2 & 15,6 & 20,4 & 18,7 & 12,0 & 39,8 & 42,0 & 27,4 \\
\hline De 36 à 45 ans & 28,5 & 29,5 & 29,9 & 33,3 & 27,4 & 28,7 & 26,8 & 33,2 & 23,4 & 42,8 & 24,4 & 29,8 \\
\hline De 46 à 60 ans & 47,6 & 34,6 & 19,6 & 40,7 & 21,0 & 52,6 & 50,3 & 45,9 & 64,3 & 13,4 & 17,8 & 34,8 \\
\hline \multicolumn{13}{|l|}{ Diplôme } \\
\hline Pas de diplôme & 0,4 & 1,1 & 3,0 & 1,7 & 0,5 & 0,4 & 0,0 & 0,0 & 0,0 & 2,3 & 0,5 & 0,3 \\
\hline Sortie du lycée sans diplôme & 3,8 & 4,9 & 11,7 & 8,7 & 0,0 & 3,2 & 1,7 & 1,8 & 1,3 & 2,0 & 1,4 & 2,4 \\
\hline Diplôme du lycée & 46,6 & 42,2 & 55,1 & 52,2 & 22,9 & 42,8 & 36,7 & 33,5 & 32,7 & 30,6 & 55,3 & 36,4 \\
\hline Associate degree $(\mathrm{Bac}+2$, professionnel) & 5,5 & 4,6 & 5,1 & 7,8 & 2,7 & 3,6 & 5,6 & 6,6 & 0,7 & 2,4 & 3,0 & 2,4 \\
\hline Associate degree (Bac +2 , académique) & 7,0 & 6,4 & 5,6 & 5,7 & 6,9 & 6,7 & 6,9 & 6,8 & 10,3 & 8,0 & 3,8 & 5,9 \\
\hline Bachelor's degree $(\mathrm{Bac}+3)$ & 24,0 & 24,4 & 14,2 & 13,5 & 40,0 & 22,6 & 31,3 & 29,5 & 26,3 & 25,1 & 23,6 & 30,2 \\
\hline Master's degree $(B a c+5)$ & 9,6 & 11,6 & 4,4 & 8,9 & 13,9 & 15,1 & 14,4 & 13,9 & 20,1 & 22,5 & 11,0 & 14,8 \\
\hline Formations professionnelles $(\mathrm{Bac}+2)$ & 1,7 & 2,9 & 0,6 & 1,3 & 9,6 & 2,8 & 1,6 & 5,4 & 3,0 & 6,1 & 0,1 & 4,2 \\
\hline Doctorat & 1,5 & 1,9 & 0,4 & 0,4 & 3,6 & 2,9 & 1,8 & 2,5 & 5,6 & 1,1 & 1,4 & 3,3 \\
\hline \multicolumn{13}{|l|}{ Type de ménage } \\
\hline Homme célibataire sans enfant & 7,4 & 6,4 & 5,2 & 8,2 & 5,0 & 6,0 & 6,6 & 4,4 & 12,3 & 10,7 & 9,3 & 7,1 \\
\hline Femme célibataire sans enfant & 7,9 & 6,4 & 6,1 & 7,9 & 3,8 & 8,8 & 5,2 & 8,7 & 6,7 & 4,5 & 4,0 & 8,4 \\
\hline Homme célibataire avec enfants & 1,5 & 1,6 & 1,7 & 1,4 & 1,0 & 1,5 & 2,0 & 0,8 & 0,6 & 0,0 & 1,0 & 4,1 \\
\hline Femme célibataire avec enfants & 4,7 & 5,8 & 8,5 & 10,4 & 1,8 & 3,2 & 5,0 & 4,2 & 1,8 & 9,6 & 7,0 & 3,3 \\
\hline Homme en couple sans enfant & 18,4 & 15,4 & 12,1 & 13,1 & 21,5 & 22,8 & 17,0 & 13,9 & 21,6 & 8,2 & 14,9 & 13,5 \\
\hline Femme en couple sans enfant & 18,8 & 16,3 & 10,5 & 14,2 & 20,9 & 17,1 & 20,3 & 16,5 & 21,1 & 13,2 & 16,9 & 21,0 \\
\hline Homme en couple avec enfants & 22,6 & 25,1 & 29,2 & 20,5 & 23,0 & 20,4 & 25,3 & 28,3 & 22,5 & 21,7 & 20,2 & 22,6 \\
\hline Femme en couple avec enfants & 18,7 & 23,1 & 26,7 & 24,4 & 23,1 & 20,1 & 18,7 & 23,3 & 13,3 & 32,3 & 26,8 & 19,9 \\
\hline \multicolumn{13}{|l|}{ Lieu de résidence } \\
\hline Habite en métropole & 81,5 & 93,8 & 93,5 & 95,4 & 96,6 & 91,5 & 90,8 & 95,8 & 89,4 & 98,4 & 99,0 & 91,9 \\
\hline Habite hors métropole & 18,5 & 6,2 & 6,5 & 4,6 & 3,4 & 8,5 & 9,3 & 4,2 & 10,7 & 1,7 & 1,0 & 8,1 \\
\hline \multicolumn{12}{|l|}{ Situation sur le marché du travail } & \\
\hline Actif occupé & 92,3 & 92,2 & 90,5 & 90,4 & 93,4 & 92,9 & 92,9 & 93,4 & 93,0 & 88,2 & 91,7 & 95,8 \\
\hline Chômeur (à la recherche d'un emploi) & 3,4 & 4,1 & 5,1 & 4,8 & 3,7 & 1,9 & 4,5 & 2,6 & 6,4 & 5,6 & 5,5 & 1,4 \\
\hline Autre chômeur & 0,7 & 0,5 & 0,7 & 0,8 & 0,3 & 0,1 & 0,3 & 0,3 & 0,0 & 0,0 & 1,2 & 0,6 \\
\hline Autre personne sans emploi & 3,6 & 3,2 & 3,6 & 4,0 & 2,6 & 5,2 & 2,3 & 3,7 & 0,6 & 6,2 & 1,7 & 2,2 \\
\hline Nombre d'observations & 42354 & 3406 & 916 & 253 & 324 & 248 & 537 & 412 & 147 & 98 & 184 & 287 \\
\hline
\end{tabular}

Lecture : Parmi les Américains dont les parents sont nés américains, 1,47\% sont titulaires d'un doctorat.

Champ : Individus de nationalité américaine, ayant entre 20 et 60 ans, vivant en logement ordinaire et qui ne sont ni étudiant, ni retraité, ni travailleur indépendant.

Source : Current Population Survey 2012, BLS.

\section{L'intégration sur le marché du travail}

L'examen des statistiques descriptives de la qualité de l'emploi met en lumière une pénalité pour les descendants d'immigrés dans l'accès à l'emploi et la plupart des dimensions de la qualité de l'emploi étudiées, à l'exception du salaire aux États-Unis.

Dans les deux pays, il existe de forts écarts d'insertion dans l'emploi selon les origines. Le tableau 1 montre en France un écart de 10 points de pourcentage entre les natifs en emploi et les descendants d'immigrés en emploi. L'écart est encore plus élevé lorsqu'il s'agit des descendants d'immigrés maghrébins, puisque les actifs occupés ne représentent que $56 \%$ d'entre eux contre $77 \%$ des natifs. Aux États-Unis, les écarts entre natifs et descendants d'immigrés sont d'ampleur moindre. La décomposition entre origines dépeint néanmoins des écarts significatifs, allant jusqu'à 4 points de pourcentage pour les descendants d'immigrés portoricains par rapport aux natifs; et contrairement au cas français, les actifs occupés descendants d'immigrés peuvent être surreprésentés dans l'emploi aux États-Unis (c'est par exemple le cas des descendants d'immigrés d'Asie, d'Europe ou du Canada). 
Tableau 3 : Les caractéristiques de l'emploi selon l'origine en France

\begin{tabular}{|c|c|c|c|c|c|c|c|}
\hline & \multirow{2}{*}{$\begin{array}{l}\text { Français } \\
\text { nés de } \\
\text { parents } \\
\text { français }\end{array}$} & \multirow[b]{2}{*}{$\begin{array}{c}\text { Enfants } \\
\text { d'immigrés }\end{array}$} & \multicolumn{5}{|c|}{ Dont } \\
\hline & & & Maghreb & $\begin{array}{l}\text { Europe } \\
\text { du Sud }\end{array}$ & $\begin{array}{l}\text { Reste de } \\
\text { l'Afrique }\end{array}$ & $\begin{array}{l}\text { Europe } \\
\text { de l'Est }\end{array}$ & $\begin{array}{l}\text { Europe } \\
\text { du Nord }\end{array}$ \\
\hline Salaire mensuel moyen en euros & 1902 & 1743 & 1629 & 1761 & 1485 & 1888 & 2036 \\
\hline Type de contrat de travail (en \%) & & & & & & & \\
\hline$C D I$ & 88,5 & 85,5 & 82,7 & 89,4 & 73,7 & 89,0 & 86,6 \\
\hline$C D D$ (ou autres contrats à durée déterminée) & 11,6 & 14,5 & 17,3 & 10,6 & 26,3 & 11,0 & 13,4 \\
\hline Temps de travail (en \%) & & & & & & & \\
\hline Temps plein & 82,2 & 81,5 & 80,9 & 83,1 & 76,5 & 83,9 & 78,7 \\
\hline Temps partiel non subi & 0,9 & 1,2 & 0,5 & 1,0 & 2,7 & 0,7 & 3,6 \\
\hline Temps partiel subi & 16,9 & 17,3 & 18,6 & 16,0 & 20,8 & 15,4 & 17,7 \\
\hline Nombre d'observations & 22505 & 2531 & 597 & 1080 & 133 & 170 & 240 \\
\hline
\end{tabular}

Lecture : Parmi les Français dont les parents sont nés français, $82,24 \%$ travaillent à temps plein.

Champ : Individus de nationalité française, ayant entre 20 et 60 ans, vivant en logement ordinaire et qui ne sont ni étudiant, ni retraité, ni travailleur indépendant.

Source : Enquête Emploi en continu 2012, Insee.

Tableau 4 : Les caractéristiques de l'emploi selon l'origine aux États-Unis

\begin{tabular}{|c|c|c|c|c|c|c|c|c|c|c|c|c|}
\hline & \multirow{2}{*}{ 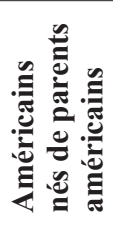 } & \multirow[b]{2}{*}{ 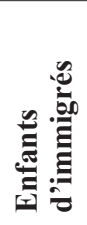 } & \multicolumn{10}{|c|}{ Dont } \\
\hline & & & 节 & 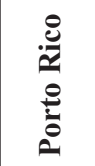 & $\frac{.0}{4}$ & U్ & 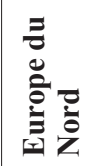 & 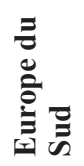 & 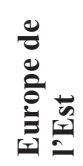 & 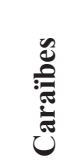 & 窇 & 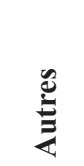 \\
\hline Salaire mensuel moyen en dollars & 4698 & 4755 & 3677 & 3464 & 5725 & 4895 & 5102 & 6500 & 5473 & 4049 & 3912 & 5204 \\
\hline Type de contrat de travail (en \%) & & & & & & & & & & & & \\
\hline Avec assurance-santé & 60,7 & 58,9 & 52,0 & 57,8 & 67,5 & 60,2 & 61,9 & 61,5 & 65,1 & 64,6 & 56,6 & 58,3 \\
\hline Sans assurance-santé & 39,3 & 41,1 & 48,0 & 42,2 & 32,5 & 39,8 & 38,1 & 38,5 & 34,9 & 35,4 & 43,4 & 41,7 \\
\hline Temps de travail (en \%) & & & & & & & & & & & & \\
\hline Temps plein & 71,1 & 70,4 & 71,8 & 64,5 & 74,9 & 66,2 & 67,9 & 70,7 & 74,0 & 72,1 & 70,0 & 72,4 \\
\hline Temps partiel non subi & 20,6 & 19,8 & 18,4 & 26,9 & 15,6 & 23,1 & 21,4 & 19,2 & 18,1 & 18,7 & 19,9 & 19,4 \\
\hline Temps partiel subi & 8,4 & 9,7 & 9,8 & 8,7 & 9,5 & 10,7 & 10,7 & 10,1 & 7,9 & 9,3 & 10,1 & 8,2 \\
\hline Nombre d'observations & 39295 & 3150 & 840 & 243 & 320 & 258 & 505 & 378 & 109 & 78 & 174 & 245 \\
\hline
\end{tabular}

Lecture : Parmi les Américains dont les parents sont nés américains, $71,1 \%$ travaillent à temps plein.

Champ : Individus de nationalité américaine, ayant entre 20 et 60 ans, vivant en logement ordinaire et qui ne sont ni étudiant, ni retraité, ni travailleur indépendant.

Source : Current Population Survey 2012, BLS.

Comme le montre le tableau 3, les descendants d'immigrés ont des salaires inférieurs en moyenne à ceux des natifs en France. On observe une fois encore une hétérogénéité très marquée entre les origines des parents. Ainsi, les descendants d'immigrés maghrébins ou d'Afrique subsaharienne ont par exemple un salaire mensuel moyen inférieur aux natifs, respectivement de 273 et $417 €$. Seuls les descendants d'immigrés d'Europe du Nord ont des salaires en moyenne supérieurs à ceux des natifs de $134 €$. Aux États-Unis, la conclusion est différente puisque les descendants d'immigrés ont dans l'ensemble des salaires supérieurs aux natifs (tableau 4). Cet écart positif peut renvoyer à des différences de durée du travail, de secteurs d'activité ou de métiers (OCDE, 2010), voire à des conditions de travail plus difficiles (suivant la théorie des différences compensatrices $\left.{ }^{(10)}\right)$. Par ailleurs, les fortes inégalités ethnocommunautaires au sein même du groupe des natifs (notamment entre les «Noirs» et les «Blancs») peuvent également expliquer cet écart positif : il pourrait ainsi voiler de fortes inégalités entre natifs selon leur couleur de peau. Pour les non-natifs, les inégalités salariales entre origines sont de fait plus marquées aux États-Unis qu'en France. Les descendants d'immigrés asiatiques ou européens ont des

(10) «La théorie des différences compensatrices indique que le libre jeu de la concurrence sur les marchés doit conduire à des différences de salaire uniquement induites par des différences de pénibilité des tâches et de compétence.» (CAHUC, 2001, p. 14.) 
salaires moyens près de deux fois plus élevés que les descendants d'immigrés hispaniques (originaires du Mexique, de Porto Rico ou d'Amérique latine). Ces fortes différences aux États-Unis peuvent en partie s'expliquer par les structures d'âges et de diplômes, plus disparates selon l'origine qu'en France (voir tableaux 1 et 2) ainsi que par les inégalités de salaires plus importantes aux États-Unis (PiketTy, Saez, 2003).

En France, 1'accès au CDI est fortement inégalitaire selon les origines. Le tableau 3 met en lumière la surreprésentation des Africains (du Maghreb ou de la zone subsaharienne) dans les emplois à durée déterminée. À l'inverse, les descendants d'immigrés d'Europe de l'Est et du Sud sont en moyenne plus en CDI que les natifs. La sécurité de l'emploi, mesurée par l'accès à l'assurance-santé obtenu par l'employeur, est en moyenne légèrement plus faible pour les descendants d'immigrés que pour les natifs aux États-Unis - de seulement deux points de pourcentage - mais l'hétérogénéité entre les origines est ici aussi marquée (voir tableau 4) : les descendants d'immigrés asiatiques sont les moins touchés puisqu'ils bénéficient globalement d'une meilleure sécurité de l'emploi que les natifs, au contraire des descendants d'immigrés mexicains par exemple.

Au niveau agrégé, les inégalités entre natifs et descendants d'immigrés en France en termes de temps de travail sont faibles (voir tableau 3). Les descendants d'immigrés d'Afrique subsaharienne ou d'Europe du Nord restent néanmoins proportionnellement plus en temps partiel subi, tandis que les descendants d'immigrés d'autres origines le sont parfois moins que les natifs (cas des descendants d'immigrés maghrébins ou d'Europe de l'Est). Aux États-Unis, le temps partiel subi touche également davantage les descendants d'immigrés, tandis que l'emploi à temps plein les concerne en moyenne moins (voir tableau 4). Les descendants d'immigrés hispaniques ou africains sont notamment parmi les plus touchés par le temps partiel subi, tandis que les descendants d'immigrés asiatiques occupent relativement plus d'emplois à temps plein que la moyenne des natifs.

\section{Une pénalité liée à l'origine sur l'accès à l'emploi et sur sa qualité}

Les statistiques descriptives ont mis en évidence de fortes inégalités entre natifs et descendants d'immigrés, puis entre descendants d'immigrés de différentes origines. L'analyse économétrique cherche à déterminer ici le rôle joué par l'origine en tenant compte de la sélection à l'entrée dans l'emploi dans chacun des deux pays, étant notamment donné la discrimination à l'embauche dont sont potentiellement victimes les descendants d'immigrés (OCDE, 2012). Un modèle d'Heckman est donc estimé avec pour conditions d'exclusion les caractéristiques du ménage. Conformément aux liens théoriques avancés dans la littérature (MrOz, 1987; Hyslop, 1999; BuCHINSKY et al., 2010), les variables d'exclusion sont significatives dans les deux pays.

\section{Le rôle de l'ascendance migratoire plus élevé en France}

Les résultats de nos estimations indiquent d'abord un effet négatif d'être descendant d'immigré sur la probabilité d'entrer en emploi en France alors que cet effet n'est pas significatif aux États-Unis. Ensuite, être descendant d'immigré en France augmente le salaire (voir tableau 5) mais affecte négativement la sécurité de l'emploi et le temps de travail. Aux États-Unis, comme en France, un coefficient positif est associé au salaire alors que les autres dimensions ne sont pas significativement influencées par l'ascendance migratoire (voir tableau 6). Un examen plus précis des variables de contrôle peut également illustrer nos hypothèses ${ }^{(11)}$.

Dans les deux pays, être une femme conduit à un effet négatif sur la qualité de l'emploi et sur la probabilité d'entrer en emploi. L'âge a, lui aussi, l'effet attendu, c'est-à-dire un effet positif à la fois sur la probabilité d' entrer en emploi et sur sa qualité ensuite. De même, les diplômes semblent avoir un effet protecteur puisque plus le diplôme est élevé, plus la probabilité d'être en emploi et la qualité de l'emploi augmentent. Le lieu de résidence est également significativement lié à l'entrée dans l'emploi et à sa qualité. En France, habiter dans une ZUS décroît la probabilité d'entrer en emploi (Fitoussi et al., 2004); les effets sur la qualité de l'emploi sont variables selon que la ZUS est en région parisienne ou non (voir tableau 5). Aux États-Unis, le fait d'habiter dans une métropole est négativement lié à la qualité de l'emploi et à la probabilité d'entrer en emploi. Ce résultat tend à confirmer les précédentes études sur la plus faible qualité des emplois dans les grandes villes ou métropoles (BERNHARDT et al., 2013). Enfin, en France, l'effet d'avoir uniquement un parent immigré est positif sur l'entrée dans l'emploi et sur le salaire, ce qui peut renvoyer à l'hypothèse d'un rôle «intégrateur» du deuxième parent natif (Algan et al., 2012). À l'inverse, aux États-Unis, l'effet de n'avoir qu'un seul parent immigré est moins net sur la qualité de l'emploi : nous trouvons un effet négatif mais faible sur le temps de travail, et aucun effet sur la probabilité d'entrer en emploi.

(11) Tous les contrôles ne sont pas reportés dans les tableaux mais sont disponibles sur demande (âge ${ }^{2}$, secteur, année). 
Tableau 5 : Estimations des déterminants de la qualité de l'emploi en France avec une correction de l'endogénéité

\begin{tabular}{|c|c|c|c|c|c|c|}
\hline \multirow[b]{2}{*}{ Variable } & \multicolumn{2}{|c|}{ Modèle 1} & \multicolumn{2}{|c|}{ Modèle 2} & \multicolumn{2}{|c|}{ Modèle 3} \\
\hline & Salaire & $\begin{array}{c}\text { Entrée dans } \\
\text { l'emploi }\end{array}$ & $\begin{array}{c}\text { Temps de } \\
\text { travail }\end{array}$ & $\begin{array}{c}\text { Entrée dans } \\
\text { l'emploi }\end{array}$ & $\begin{array}{c}\text { Sécurité de } \\
\text { l'emploi }\end{array}$ & $\begin{array}{c}\text { Entrée dans } \\
\text { l'emploi }\end{array}$ \\
\hline Non-issu de l'immigration & Ref. & Ref. & Ref. & Ref. & Ref. & Ref. \\
\hline Descendant d'immigré & $\begin{array}{r}0,027 * * * \\
(-0,007)\end{array}$ & $\begin{array}{r}-0,27 * * * \\
(-0,02)\end{array}$ & $\begin{array}{r}-0,17 * * * \\
(-0,02)\end{array}$ & $\begin{array}{r}-0,32 * * * \\
(-0,02)\end{array}$ & $\begin{array}{r}-0,06^{* *} \\
(-0,03)\end{array}$ & $\begin{array}{r}-0,31 * * * \\
(-0,02)\end{array}$ \\
\hline Être une femme & $\begin{array}{r}-0,221^{* * *} \\
(-0,003)\end{array}$ & $\begin{array}{r}-0,21 * * * \\
(-0,01)\end{array}$ & $\begin{array}{r}-0,83 * * * \\
(-0,02)\end{array}$ & $\begin{array}{r}-0,30 * * * \\
(-0,01)\end{array}$ & $\begin{array}{r}-0,09 * * * \\
(-0,01)\end{array}$ & $\begin{array}{r}-0,29 * * * \\
(-0,01)\end{array}$ \\
\hline Âge & $\begin{array}{r}0,047 * * * \\
(-0,001)\end{array}$ & $\begin{array}{r}0,01 * * * \\
(0,00)\end{array}$ & $\begin{array}{r}0,05 * * * \\
(0,00)\end{array}$ & $\begin{array}{r}0,01 * * * \\
(0,00)\end{array}$ & $\begin{array}{r}0,19 * * * \\
(0,00)\end{array}$ & $\begin{array}{r}0,01 * * * \\
(0,00)\end{array}$ \\
\hline Sans diplôme & $\begin{array}{r}-0,209 * * * \\
(-0,005)\end{array}$ & $\begin{array}{r}-0,40 * * * \\
(-0,01)\end{array}$ & $\begin{array}{r}-0,38 * * * \\
(-0,01)\end{array}$ & $\begin{array}{r}-0,46 * * * \\
(-0,01)\end{array}$ & $\begin{array}{r}-0,19 * * * \\
(-0,02)\end{array}$ & $\begin{array}{r}-0,45^{* * *} \\
(-0,01)\end{array}$ \\
\hline Brevet des collèges/BEPC & $\begin{array}{r}-0,095 * * * \\
(-0,006)\end{array}$ & $\begin{array}{r}-0,14 * * * \\
(-0,01)\end{array}$ & $\begin{array}{r}-0,14 * * * \\
(-0,02)\end{array}$ & $\begin{array}{r}-0,16 * * * \\
(-0,01)\end{array}$ & $\begin{array}{r}-0,10 * * * \\
(-0,02)\end{array}$ & $\begin{array}{r}-0,16^{* * *} \\
(-0,01)\end{array}$ \\
\hline $\mathrm{BEP} / \mathrm{CAP}$ & $\begin{array}{r}-0,156^{* * *} \\
(-0,004)\end{array}$ & $\begin{array}{r}0,03 * * * \\
(-0,01)\end{array}$ & $\begin{array}{r}0,01 \\
(-0,01)\end{array}$ & $\begin{array}{r}0,04 * * * \\
(-0,01)\end{array}$ & $\begin{array}{r}-0,08 * * * \\
(-0,02)\end{array}$ & $\begin{array}{r}0,05 * * * \\
(-0,01)\end{array}$ \\
\hline Baccalauréat & Ref. & Ref. & Ref. & Ref. & Ref. & Ref. \\
\hline $\mathrm{Bac}+2$ & $\begin{array}{r}0,092 * * * \\
(-0,005)\end{array}$ & $\begin{array}{r}0,30 * * * \\
(-0,01)\end{array}$ & $\begin{array}{r}0,25 * * * \\
(-0,01)\end{array}$ & $\begin{array}{r}0,36 * * * \\
(-0,01)\end{array}$ & $\begin{array}{r}0,09 * * * \\
(-0,02)\end{array}$ & $\begin{array}{r}0,37 * * * \\
(-0,01)\end{array}$ \\
\hline Licence ou plus & $\begin{array}{r}0,294 * * * \\
(-0,005)\end{array}$ & $\begin{array}{r}0,33 * * * \\
(-0,01)\end{array}$ & $\begin{array}{r}0,28 * * * \\
(-0,01)\end{array}$ & $\begin{array}{r}0,34 * * * \\
(-0,01)\end{array}$ & $\begin{array}{r}0,09 * * * \\
(-0,02)\end{array}$ & $\begin{array}{r}0,33 * * * \\
(-0,01)\end{array}$ \\
\hline Habiter en province hors d'une ZUS & Ref. & Ref. & Ref. & Ref. & Ref. & Ref. \\
\hline Habiter en région parisienne hors d'une ZUS & $\begin{array}{r}0,169 * * * \\
(-0,004)\end{array}$ & $\begin{array}{r}0,08 * * * \\
(-0,01)\end{array}$ & $\begin{array}{r}0,24 * * * \\
(-0,01)\end{array}$ & $\begin{array}{r}0,08 * * * \\
(-0,01)\end{array}$ & $\begin{array}{r}0,15^{* * *} \\
(-0,02)\end{array}$ & $\begin{array}{r}0,09 * * * \\
(-0,01)\end{array}$ \\
\hline Habiter en province dans une ZUS & $\begin{array}{r}0,008 \\
(-0,009)\end{array}$ & $\begin{array}{r}-0,24 * * * \\
(-0,02)\end{array}$ & $\begin{array}{r}-0,18 * * * \\
(-0,02)\end{array}$ & $\begin{array}{r}-0,26 * * * \\
(-0,02)\end{array}$ & $\begin{array}{r}-0,09 * * * \\
(-0,03)\end{array}$ & $\begin{array}{r}-0,25 * * * \\
(-0,02)\end{array}$ \\
\hline Habiter en région parisienne dans une ZUS & $\begin{array}{r}0,078 * * * \\
(-0,016)\end{array}$ & $\begin{array}{l}-0,06^{*} \\
(-0,03)\end{array}$ & $\begin{array}{r}0,18 * * * \\
(-0,05)\end{array}$ & $\begin{array}{r}-0,05 \\
(-0,04)\end{array}$ & $\begin{array}{r}0,08 \\
(-0,06)\end{array}$ & $\begin{array}{r}-0,04 \\
(-0,04)\end{array}$ \\
\hline Avoir un seul parent immigré & $\begin{array}{l}0,021 * * \\
(-0,010)\end{array}$ & $\begin{array}{r}0,02 \\
(-0,02) \\
\text { Ref }\end{array}$ & $\begin{array}{r}0,00 \\
(-0,03)\end{array}$ & $\begin{array}{r}0,04 * \\
(-0,02) \\
\operatorname{Ref}\end{array}$ & $\begin{array}{r}0,04 \\
(-0,03)\end{array}$ & $\begin{array}{r}0,02 \\
(-0,02) \\
\text { Ref }\end{array}$ \\
\hline Ne pas avoir d'enfant & & & & Rer. & & Ker. \\
\hline Avoir un enfant & & $\begin{array}{r}0,04 * * * \\
(-0,01)\end{array}$ & & $\begin{array}{r}0,10 * * * \\
(-0,01)\end{array}$ & & $\begin{array}{r}0,08 * * * \\
(-0,01)\end{array}$ \\
\hline Deux enfants & & $\begin{array}{r}0,09 * * * \\
(-0,01)\end{array}$ & & $\begin{array}{r}0,23 * * * \\
(-0,01)\end{array}$ & & $\begin{array}{r}0,16^{* * * *} \\
(-0,01)\end{array}$ \\
\hline Trois enfants & & $\begin{array}{r}-0,14 * * * \\
(-0,01)\end{array}$ & & $\begin{array}{r}0,02 \\
(-0,01)\end{array}$ & & $\begin{array}{r}-0,17 * * * \\
(-0,01)\end{array}$ \\
\hline Être en couple & & $\begin{array}{r}0,18^{* * *} \\
(-0,01)\end{array}$ & & $\begin{array}{r}0,18^{* * *} \\
(-0,01)\end{array}$ & & $\begin{array}{r}0,23 * * * \\
(-0,01)\end{array}$ \\
\hline Constante & $\begin{array}{r}6,437 * * * \\
(-0,019)\end{array}$ & $\begin{array}{r}0,42 * * * \\
(-0,02)\end{array}$ & $\begin{array}{r}-0,09 \\
(-0,06) \\
\end{array}$ & $\begin{array}{r}0,38 * * * \\
(-0,02)\end{array}$ & $\begin{array}{r}2,50 * * * \\
(-0,08)\end{array}$ & $\begin{array}{r}0,44 * * * \\
(-0,02) \\
\end{array}$ \\
\hline $\begin{array}{l}\text { Rho } \\
\text { Observations }\end{array}$ & $\begin{array}{r}0,846^{* * * *} \\
116459\end{array}$ & 153422 & $\begin{array}{r}0,845 * * * \\
116440\end{array}$ & 153422 & $\begin{array}{r}0,451 * * * \\
116440\end{array}$ & 153422 \\
\hline
\end{tabular}

Variables contrôlées disponibles sur demande : âge², secteur d'activité, année.

Notes : La régression sur le salaire est linéaire (MCO), les régressions sur le temps de travail et sur la sécurité de l'emploi sont des probits binomiaux. Les symboles *, ** et *** représentent les seuils de significativité statistique à respectivement 10,5 et $1 \%$.

Champ : Natifs et descendants d'immigrés en emploi, de 20 à 60 ans, en France métropolitaine.

Source : Enquêtes Emploi en continu 2008-2012, Insee. 
Tableau 6 : Estimations des déterminants de la qualité de l'emploi aux États-Unis avec une correction de l'endogénéité

\begin{tabular}{|c|c|c|c|c|c|c|}
\hline \multirow[b]{2}{*}{ Variable } & \multicolumn{2}{|c|}{ Modèle 1} & \multicolumn{2}{|c|}{ Modèle 2} & \multicolumn{2}{|c|}{ Modèle 3} \\
\hline & Salaire & $\begin{array}{c}\text { Entrée dans } \\
\text { l'emploi }\end{array}$ & $\begin{array}{l}\text { Temps de } \\
\text { travail }\end{array}$ & $\begin{array}{c}\text { Entrée dans } \\
\text { l'emploi }\end{array}$ & $\begin{array}{l}\text { Sécurité de } \\
\text { l'emploi }\end{array}$ & $\begin{array}{l}\text { Entrée dans } \\
\text { l'emploi }\end{array}$ \\
\hline Non-issu de l'immigration & Ref. & Ref. & Ref. & Ref. & Ref. & Ref. \\
\hline \multirow[t]{2}{*}{ Descendant d'immigré } & $0,040 * * *$ & 0,01 & 0,00 & 0,02 & 0,00 & 0,03 \\
\hline & $(-0,010)$ & $(-0,02)$ & $(-0,02)$ & $(-0,02)$ & $(-0,02)$ & $(-0,02)$ \\
\hline \multirow[t]{2}{*}{ Être une femme } & $-0,483 * * *$ & $-0,05 * * *$ & $-0,45 * * *$ & $-0,10 * * *$ & $-0,40 * * *$ & $-0,12 * * *$ \\
\hline & $(-0,004)$ & $(-0,01)$ & $(-0,01)$ & $(-0,01)$ & $(-0,01)$ & $(-0,01)$ \\
\hline \multirow[t]{2}{*}{ Âge } & $0,068 * * *$ & $0,01 * * *$ & $0,04 * * *$ & $0,01 * * *$ & $0,02 * * *$ & $0,01 * * *$ \\
\hline & $(-0,001)$ & $(0,00)$ & $(0,00)$ & $(0,00)$ & $(0,00)$ & $(0,00)$ \\
\hline \multirow[t]{2}{*}{ Sans diplôme } & $-0,390 * * *$ & $-0,17 * * *$ & $-0,35 * * *$ & $-0,30 * * *$ & $-0,52 * * *$ & $-0,29 * * *$ \\
\hline & $(-0,025)$ & $(-0,04)$ & $(-0,05)$ & $(-0,04)$ & $(-0,04)$ & $(-0,04)$ \\
\hline \multirow[t]{2}{*}{ Sortie du lycée en fin de cycle sans diplôme } & $-0,230 * * *$ & $-0,28 * * *$ & $-0,20 * * *$ & $-0,37 * * *$ & $-0,41 * * *$ & $-0,36 * * *$ \\
\hline & $(-0,010)$ & $(-0,02)$ & $(-0,02)$ & $(-0,02)$ & $(-0,01)$ & $(-0,02)$ \\
\hline Diplôme du lycée (éq. Bac) & Ref. & Ref. & Ref. & Ref. & Ref. & Ref. \\
\hline \multirow[t]{2}{*}{$\mathrm{Bac}+2$} & $0,151 * * *$ & $0,16^{* * *}$ & 0,02 & $0,20 * * *$ & $0,14 * * *$ & $0,19 * * *$ \\
\hline & $(-0,006)$ & $(-0,01)$ & $(-0,01)$ & $(-0,01)$ & $(-0,01)$ & $(-0,01)$ \\
\hline \multirow[t]{2}{*}{ Licence ou plus } & $0,490 * * *$ & $0,39 * * *$ & $0,20 * * *$ & $0,37 * * *$ & $0,31 * * *$ & $0,35 * * *$ \\
\hline & $(-0,004)$ & $(-0,01)$ & $(-0,01)$ & $(-0,01)$ & $(-0,01)$ & $(-0,01)$ \\
\hline \multirow[t]{2}{*}{ Habiter dans une métropole } & $-0,181 * * *$ & $-0,02 *$ & $-0,07 * * *$ & $-0,01$ & $-0,05 * * *$ & $-0,01$ \\
\hline & $(-0,004)$ & $(-0,01)$ & $(-0,01)$ & $(-0,01)$ & $(-0,01)$ & $(-0,01)$ \\
\hline \multirow[t]{2}{*}{ Avoir un seul parent immigré } & 0,000 & 0,02 & $0,07 * *$ & 0,01 & 0,00 & 0,01 \\
\hline & $(-0,013)$ & $(-0,03)$ & $(-0,03)$ & $(-0,03)$ & $(-0,02)$ & $(-0,03)$ \\
\hline Ne pas avoir d'enfant & & Ref. & & Ref. & & Ref. \\
\hline \multirow[t]{2}{*}{ Avoir un enfant } & & $0,08 * * *$ & & $0,07 * * *$ & & $0,08 * * *$ \\
\hline & & $(-0,01)$ & & $(-0,01)$ & & $(-0,01)$ \\
\hline \multirow[t]{2}{*}{ Deux enfants } & & $0,13 * * *$ & & $0,11 * * *$ & & $0,14 * * *$ \\
\hline & & $(-0,01)$ & & $(-0,01)$ & & $(-0,01)$ \\
\hline \multirow[t]{2}{*}{ Trois enfants } & & $0,06^{* * *}$ & & 0,00 & & $0,06^{* * *}$ \\
\hline & & $(-0,01)$ & & $(-0,01)$ & & $(-0,01)$ \\
\hline \multirow[t]{2}{*}{ Être en couple } & & $0,19 * * *$ & & $0,20 * * *$ & & $0,27 * * *$ \\
\hline & & $(-0,01)$ & & $(-0,01)$ & & $(-0,01)$ \\
\hline \multirow[t]{2}{*}{ Constante } & $-13,86^{* * *}$ & 7,54 & $69,86 * * *$ & $14,47 * * *$ & $37,61 * * *$ & $9,53 *$ \\
\hline & $(-2,484)$ & $(-5,09)$ & $(-5,94)$ & $(-5,43)$ & $(-3,74)$ & $(-5,41)$ \\
\hline Rho & $-0,848 * * *$ & & $-0,722 * * *$ & & $-0,932 * * *$ & \\
\hline Observations & 153422 & 244701 & 153422 & 244701 & 153422 & 244701 \\
\hline
\end{tabular}

Variables contrôlées disponibles sur demande : âge², secteur d'activité, année.

Notes : La régression sur le salaire est linéaire (MCO), les régressions sur le temps de travail et sur la sécurité de l'emploi sont des probits binomiaux. Les symboles *, ${ }^{*}$ et $* * *$ représentent les seuils de significativité statistique à respectivement 10,5 et $1 \%$.

Champ : Natifs et descendants d'immigrés en emploi, de 20 à 60 ans, aux États-Unis.

Source: Current Population Surveys 2008-2012, BLS. 


\section{L'hétérogénéité entre origines}

Compte tenu de l'hétérogénéité selon l'origine mise en évidence dans la littérature, la variable descendant d'immigré a été, dans une seconde étape, désagrégée par origine. Cette désagrégation révèle d'abord en France un effet négatif de toutes les origines sur la probabilité d'entrer dans l'emploi sauf pour les descendants d'immigrés d'Europe du Sud, pour lesquels aucun effet significatif ne peut être relevé. Être descendant d'immigré africain décroît ainsi d'environ $35 \%$ la probabilité d'entrer en emploi, tandis que ce pourcentage avoisine les $15 \%$ pour les descendants d'immigrés d'Europe de l'Est ou du Nord. De plus, la pénalité liée à l'origine est plus importante dans l'accès à l'emploi que sur sa qualité (voir tableau 7). Les descendants d'immigrés maghrébins, après une sélection significative, ont un effet négatif lié à leur origine de $14 \%$ sur la dimension sécurité de leur emploi et de $23 \%$ sur le temps de travail, tandis qu'un effet positif est trouvé sur le salaire. Ce résultat peut s'expliquer par la plus forte sélection à l'entrée dans l'emploi de ce groupe. Aucun effet négatif lié à l'origine n'apparaît sur le salaire sauf pour les descendants d'immigrés d'Europe du Sud qui sont par ailleurs les seuls à ne pas subir de sélection négative et significative à l'entrée dans l'emploi.

À l'inverse, aux États-Unis, aucun coefficient n'est significatif à l'entrée dans l'emploi, à part pour les descendants d'immigrés caribéens et d'Europe de l'Est, avec des effets de sens opposés pour ces deux populations (un effet négatif d'environ $25 \%$ pour les premiers et positif avoisinant $22 \%$ pour les seconds). On relève des effets positifs, principalement sur le salaire, pour les différentes dimensions de la qualité de l'emploi. Ainsi, ce sont plutôt pour les descendants des vagues d'immigration non hispanophones que l'on obtient des effets positifs sur le salaire (Asie, Canada, Europe); toutefois, ils restent plus faibles que ceux relevés en France puisqu'ils sont compris entre 8 et $14 \%$. Quelques effets positifs sont notables sur la sécurité de l'emploi des descendants d'immigrés portoricains et asiatiques mais restent faibles (i. e. compris entre 6 et $8 \%$ ). Enfin, être descendant d'immigrés caribéens accroît de $28 \%$ la probabilité d'occuper un emploi à temps plein.

Dans l'ensemble, ces résultats confirment l'hétérogénéité de la situation sur le marché du travail et de la qualité de l'emploi au sein des descendants d'immigrés dans les deux pays, au-delà d'inégalités selon l'origine, globalement plus marquées en France(12). Cette hétérogénéité prend toutefois des formes bien

(12) Peu de changements sont observables sur les variables de contrôle (tableau 1 et 2 en annexe). En France, l'effet «intégrateur» dans l'emploi du fait d'avoir un parent non immigré se reporte sur l'entrée dans l'emploi. Aux Etats-Unis, seul le coefficient négatif associé au fait d'avoir un seul parent immigré se déplace du temps de travail vers le salaire lorsqu'on désagrège. différentes dans les deux pays : elle témoigne d'une forte sélection dans l'accès à l'emploi en France alors qu'elle se traduit davantage en termes de qualité (notamment salariale) aux États-Unis.

\section{Discussion des liens entre institutions et inégalités liées à l'origine}

Pour interpréter ces résultats, on peut faire l'hypothèse d'une influence des différents ensembles institutionnels des deux pays, d'une part, sur les niveaux et les écarts d'accès à l'emploi, d'autre part, sur la qualité de l'emploi.

\section{Le rôle potentiel des institutions du marché du travail dans les niveaux et les écarts de qualité de l'emploi}

La littérature sur les différentes variétés de capitalisme (GAllie, 2007; AmABLE, 2005) met en avant le rôle des institutions dans la détermination des niveaux et des écarts de qualité de l'emploi. Plus précisément, Duncan Gallie (2007) prédit une qualité de l'emploi plus faible dans les pays dits libéraux comme les États-Unis; les écarts selon les caractéristiques individuelles y seraient moindres que dans les pays continentaux comme la France, qui assureraient certes un niveau de qualité de l'emploi plus élevé mais s'accompagnant d'inégalités plus marquées. Dans cette perspective, les institutions du marché du travail influençant les différentes dimensions de la qualité de l'emploi sont multiples.

En premier lieu, les modèles français et américains se différencient par le niveau de centralisation des négociations collectives et par le taux de couverture conventionnelle, qui est nettement plus élevé en France qu'aux États-Unis (ILO, IILS, 2008). L'existence d'un salaire minimum également plus élevé en France qu'aux États-Unis implique une meilleure rémunération des emplois peu qualifiés et, en association avec la couverture conventionnelle, globalement de meilleurs salaires. En raison de la part relativement importante des peu diplômés au sein des descendants d'immigrés (Alba, HoldawAY, 2014), le salaire minimum peut permettre de réduire l'écart salarial avec les natifs. Toutefois, ces hauts niveaux induisent un coût du travail plus élevé pour les employeurs qui peuvent être plus réticents à embaucher (CAHUC, KRAMARZ, 2004; ABOwD et al., 2000). In fine, on peut penser que ces institutions françaises vont accroître la sélection à l'entrée dans l'emploi des descendants d'immigrés tout en permettant une réduction de l'écart salarial.

Les deux pays présentent ensuite des différences en termes de protection de l'emploi, qui auront des répercussions sur la sécurité de l'emploi. Selon l'OCDE, le niveau global de protection de l'emploi est plus élevé en France qu'aux États-Unis; de plus, la France se distingue de l'ensemble des pays de l'OCDE par un niveau élevé de protection des emplois temporaires (OCDE, 2013a). 
Tableau 7 : Estimations des déterminants de la qualité de l'emploi selon l'origine en France avec une correction de l'endogénéité

\begin{tabular}{|c|c|c|c|c|c|c|}
\hline \multirow[b]{2}{*}{ Variable } & \multicolumn{2}{|c|}{ Modèle 1} & \multicolumn{2}{|c|}{ Modèle 2} & \multicolumn{2}{|c|}{ Modèle 3} \\
\hline & Salaire & $\begin{array}{c}\text { Entrée dans } \\
\text { l'emploi }\end{array}$ & $\begin{array}{l}\text { Temps de } \\
\text { travail }\end{array}$ & $\begin{array}{c}\text { Entrée dans } \\
\text { l'emploi }\end{array}$ & $\begin{array}{c}\text { Sécurité de } \\
\text { l'emploi }\end{array}$ & $\begin{array}{l}\text { Entrée dans } \\
\text { l'emploi }\end{array}$ \\
\hline Non-issu de l'immigration & Ref. & Ref. & Ref. & Ref. & Ref. & Ref. \\
\hline Maghreb & $\begin{array}{r}0,034 * * * \\
(-0,010)\end{array}$ & $\begin{array}{r}-0,32 * * * \\
(-0,02)\end{array}$ & $\begin{array}{r}-0,23 * * * \\
(-0,03)\end{array}$ & $\begin{array}{r}-0,37 * * * \\
(-0,02)\end{array}$ & $\begin{array}{r}-0,14 * * * \\
(-0,03)\end{array}$ & $\begin{array}{r}-0,35 * * * \\
(-0,02)\end{array}$ \\
\hline Europe du Sud & $\begin{array}{r}-0,028 * * * \\
(-0,009)\end{array}$ & $\begin{array}{r}0,01 \\
(-0,02)\end{array}$ & $\begin{array}{r}0,04 \\
(-0,03)\end{array}$ & $\begin{array}{r}0,01 \\
(-0,02)\end{array}$ & $\begin{array}{r}-0,01 \\
(-0,03)\end{array}$ & $\begin{array}{r}0,01 \\
(-0,02)\end{array}$ \\
\hline Reste de l'Afrique & $\begin{array}{r}-0,023 \\
(-0,021)\end{array}$ & $\begin{array}{r}-0,33 * * * \\
(-0,04)\end{array}$ & $\begin{array}{r}-0,33 * * * \\
(-0,05)\end{array}$ & $\begin{array}{r}-0,41 * * * \\
(-0,04)\end{array}$ & $\begin{array}{r}-0,05 \\
(-0,07)\end{array}$ & $\begin{array}{r}-0,38 * * * \\
(-0,04)\end{array}$ \\
\hline Europe de l'Est & $\begin{array}{r}0,010 \\
(-0,019)\end{array}$ & $\begin{array}{r}-0,14 * * * \\
(-0,04)\end{array}$ & $\begin{array}{l}-0,10^{*} \\
(-0,05)\end{array}$ & $\begin{array}{r}-0,18^{* * * *} \\
(-0,05)\end{array}$ & $\begin{array}{r}-0,11 \\
(-0,07)\end{array}$ & $\begin{array}{r}-0,18^{* * *} \\
(-0,05)\end{array}$ \\
\hline Europe du Nord & $\begin{array}{r}0,006 \\
(-0,018)\end{array}$ & $\begin{array}{r}-0,13 * * * \\
(-0,04)\end{array}$ & $\begin{array}{r}-0,11 * * \\
(-0,05)\end{array}$ & $\begin{array}{r}-0,15 * * * \\
(-0,04)\end{array}$ & $\begin{array}{l}-0,11^{*} \\
(-0,06)\end{array}$ & $\begin{array}{r}-0,16 * * * \\
(-0,04)\end{array}$ \\
\hline Constante & $\begin{array}{r}6,434 * * * \\
(-0,019)\end{array}$ & $\begin{array}{r}0,44 * * * \\
(-0,02)\end{array}$ & $\begin{array}{l}-0,11^{*} \\
(-0,06)\end{array}$ & $\begin{array}{r}0,39 * * * \\
(-0,02)\end{array}$ & $\begin{array}{r}-2,51 * * * \\
(-0,08)\end{array}$ & $\begin{array}{r}0,46^{* * *} \\
(-0,02)\end{array}$ \\
\hline $\begin{array}{l}\text { Rho } \\
\text { Observations }\end{array}$ & $\begin{array}{r}-0,845 * * * \\
144591\end{array}$ & 152472 & $\begin{array}{r}0,864 * * * \\
144591\end{array}$ & 152472 & $\begin{array}{r}-0,443 * * * \\
144591\end{array}$ & 152472 \\
\hline
\end{tabular}

Variables contrôlées disponibles en annexe : voir tableau A1.

Notes : La régression sur le salaire est linéaire (MCO), les régressions sur le temps de travail et sur la sécurité de l'emploi sont des probits binomiaux. Les symboles *, ** et *** représentent les seuils de significativité statistique à respectivement 10,5 et $1 \%$.

Champ : Natifs et descendants d'immigrés en emploi, de 20 à 60 ans, en France métropolitaine.

Source : Enquêtes Emploi en continu 2008-2012, Insee.

Tableau 8 : Estimations des déterminants de la qualité de l'emploi selon l'origine aux États-Unis avec une correction de l'endogénéité

\begin{tabular}{|c|c|c|c|c|c|c|}
\hline \multirow[b]{2}{*}{ Variable } & \multicolumn{2}{|c|}{ Modèle 1} & \multicolumn{2}{|c|}{ Modèle 2} & \multicolumn{2}{|c|}{ Modèle 3} \\
\hline & Salaire & \begin{tabular}{c|} 
Entrée dans \\
l'emploi
\end{tabular} & $\begin{array}{c}\text { Temps de } \\
\text { travail }\end{array}$ & $\begin{array}{c}\text { Entrée dans } \\
\text { l'emploi }\end{array}$ & $\begin{array}{c}\text { Sécurité de } \\
\text { l'emploi }\end{array}$ & $\begin{array}{l}\text { Entrée dans } \\
\text { l'emploi }\end{array}$ \\
\hline \multirow{3}{*}{$\begin{array}{l}\text { Non-issu de l'immigration } \\
\text { Mexique }\end{array}$} & Ref. & Ref. & Ref. & Ref. & Ref. & Ref. \\
\hline & $-0,010$ & 0,00 & 0,03 & 0,03 & $-0,01$ & 0,04 \\
\hline & $(-0,014)$ & $(-0,03)$ & $(-0,03)$ & $(-0,03)$ & $(-0,02)$ & $(-0,03)$ \\
\hline \multirow[t]{2}{*}{ Porto Rico } & $-0,020$ & 0,01 & 0,01 & 0,07 & $0,06^{*}$ & 0,07 \\
\hline & $(-0,024)$ & $(-0,05)$ & $(-0,06)$ & $(-0,05)$ & $(-0,04)$ & $(-0,05)$ \\
\hline \multirow[t]{2}{*}{ Asie } & $0,144 * * *$ & 0,05 & $-0,03$ & 0,04 & $0,06^{*}$ & 0,04 \\
\hline & $(-0,023)$ & $(-0,05)$ & $(-0,06)$ & $(-0,05)$ & $(-0,04)$ & $(-0,05)$ \\
\hline \multirow[t]{2}{*}{ Canada } & $0,065^{* *}$ & 0,06 & $-0,05$ & 0,08 & $-0,02$ & 0,05 \\
\hline & $(-0,025)$ & $(-0,05)$ & $(-0,06)$ & $(-0,06)$ & $(-0,04)$ & $(-0,06)$ \\
\hline \multirow{2}{*}{ Europe du Nord } & $0,078 * * *$ & 0,03 & 0,01 & 0,01 & $-0,05$ & 0,01 \\
\hline & $(-0,020)$ & $(-0,04)$ & $(-0,05)$ & $(-0,05)$ & $(-0,03)$ & $(-0,05)$ \\
\hline \multirow[t]{2}{*}{ Europe du Sud } & $0,129 * * *$ & 0,04 & $-0,08$ & 0,00 & 0,03 & $-0,01$ \\
\hline & $(-0,021)$ & $(-0,04)$ & $(-0,05)$ & $(-0,05)$ & $(-0,03)$ & $(-0,05)$ \\
\hline \multirow[t]{2}{*}{ Europe de l'Est } & $0,098 * * *$ & 0,13 & $-0,01$ & $0,22 * *$ & $-0,07$ & $0,22 * *$ \\
\hline & $(-0,033)$ & $(-0,08)$ & $(-0,08)$ & $(-0,09)$ & $(-0,05)$ & $(-0,09)$ \\
\hline \multirow[t]{2}{*}{ Caraïbes } & 0,071 & $-0,25 * * *$ & $0,28 * *$ & $-0,28 * * *$ & $-0,08$ & $-0,25 * * *$ \\
\hline & $(-0,044)$ & $(-0,07)$ & $(-0,12)$ & $(-0,08)$ & $(-0,06)$ & $(-0,08)$ \\
\hline \multirow[t]{2}{*}{ Amérique latine } & 0,015 & $-0,01$ & 0,02 & 0,00 & $-0,07$ & 0,00 \\
\hline & $(-0,032)$ & $(-0,06)$ & $(-0,08)$ & $(-0,07)$ & $(-0,05)$ & $(-0,07)$ \\
\hline \multirow[t]{2}{*}{ Autres } & $0,078 * * *$ & 0,04 & $-0,11^{*}$ & 0,05 & $-0,08 * *$ & 0,06 \\
\hline & $(-0,026)$ & $(-0,05)$ & $(-0,06)$ & $(-0,06)$ & $(-0,04)$ & $(-0,06)$ \\
\hline \multirow[t]{2}{*}{ Constante } & $-13,920 * * *$ & 7,45 & $69,95 * * *$ & $14,43 * * *$ & $37,63 * * *$ & $9,49 *$ \\
\hline & $(-2,483)$ & $(-5,09)$ & $(-5,95)$ & $(-5,43)$ & $(-3,74)$ & $(-5,41)$ \\
\hline Rho & $-0,848 * * *$ & & $-0,725^{* * *}$ & & $0,932 * * *$ & \\
\hline Observations & 153422 & 244701 & 153422 & 244701 & 153422 & 244701 \\
\hline
\end{tabular}

Variables contrôlées disponibles en annexe : voir tableau A2.

Notes : La régression sur le salaire est linéaire (MCO), les régressions sur le temps de travail et sur la sécurité de l'emploi sont des probits binomiaux. Les symboles *, ** et *** représentent les seuils de significativité statistique à respectivement 10,5 et $1 \%$.

Champ : Natifs et descendants d'immigrés en emploi, de 20 à 60 ans, aux États-Unis.

Source : Current Population Surveys 2008-2012, BLS. 
Cette protection concourt à la meilleure qualité de l'emploi pour les salariés permanents en France mais, selon les approches néoclassiques, peut s'accompagner d'un frein sur les créations d'emplois : les ruptures d'emplois étant plus difficiles, les embauches seraient plus coûteuses pour les employeurs (CAHUC, KRAmarz, 2004). Dans un marché du travail dual, les premiers touchés sont les peu diplômés, au sein desquels on compte de nombreux descendants d'immigrés (AlbA, Holdaway, 2014). Aux États-Unis, la protection de l'emploi est plus souple pour l'ensemble des contrats, ce qui contribue à diminuer mécaniquement la qualité de l'emploi mais peut faciliter les embauches. Cette plus faible protection pour l'ensemble des contrats peut favoriser une diminution des écarts de qualité de l'emploi entre travailleurs. En France, les différences de sécurité de l'emploi entre les contrats à durée déterminée et indéterminée peuvent, à l'inverse, renforcer les inégalités de qualité de l'emploi entre travailleurs - nos résultats tendent à valider cette hypothèse.

Pour finir, les différences institutionnelles entre travailleurs à temps partiel et à temps complet peuvent accentuer les inégalités de qualité de l'emploi entre travailleurs. L'accès à des mesures de formation continue n'est ainsi pas toujours assuré aux travailleurs à temps partiel, dans les pays libéraux par exemple. De même, l'indemnisation chômage n'est effective qu'à partir de 610 heures travaillées sur les 28 derniers mois en France ${ }^{(13)}$, ce qui défavorise les salariés à temps partiel.

Dans l'ensemble, les institutions du marché du travail françaises semblent assurer une meilleure qualité de l'emploi, en particulier grâce à leur rôle protecteur sur le salaire, par rapport aux institutions américaines - nos résultats mettent en effet en lumière des écarts de salaire entre les natifs et les différents groupes de descendants d'immigrés plus importants aux États-Unis -, tout en favorisant les écarts au sein de l'emploi, notamment selon le type de contrat de travail. Les institutions françaises tendent par ailleurs à accroître les inégalités entre les individus en emploi, d'une part, et les inactifs ou les chômeurs, d'autre part, en rendant l'accès à l'emploi plus contraignant (voir les résultats du modèle de sélection). Dès lors, les inégalités qui découlent des institutions du marché du travail diffèrent selon les pays, avec plus de chômage en France et davantage de travailleurs pauvres aux États-Unis.

(13) «Au cours des 28 mois qui précèdent la fin du contrat de travail (terme du préavis) pour les moins de 50 ans [ou des] 36 mois qui précèdent la fin du contrat de travail (terme du préavis) pour les 50 ans et plus» (site de Pôle emploi : http:// www.pole-emploi.fr/candidat/les-conditions-d-attribution-de1-aide-au-retour-a-l-emploi-@/article.jspz?id=60580; consulté le 24 août 2015).

\section{Une prise en compte plus large des institutions : le cas des descendants d'immigrés}

La littérature sur les différentes variétés de capitalisme plaide toutefois pour une prise en compte plus large des institutions lorsque l'on s'intéresse à la qualité de 1'emploi (GALlie, 2007). Plus spécifiquement, considérer les descendants d'immigrés implique d'examiner l'effet des politiques d'immigration. Ces inégalités selon l'origine font ainsi écho à des politiques de sélection migratoire différentes dans les deux pays (WeIL, 2003). Aux États-Unis, les niveaux de diplômes des immigrés sont historiquement plus élevés qu'en France (DOOMERNIK et al., 2009; WeIL, 2003). La sélection accrue de l'immigration selon des critères de capital humain a pour conséquence une transmission intergénérationnelle de capital (humain, économique) plus importante pour les populations sélectionnées au titre de l'immigration du travail et une potentielle réduction des inégalités selon l'origine. La relativement meilleure intégration sur le marché du travail de certains groupes de descendants d'immigrés par rapport aux natifs aux États-Unis, mise en évidence dans notre analyse, pourrait s'expliquer ainsi.

Pour contrecarrer les niveaux plus faibles d'éducation et de capital humain de certains mouvements d'immigration, l'institution scolaire est susceptible de jouer un rôle redistributif et de réduction des inégalités liées à l'origine. En France, l'école laïque, gratuite et obligatoire, propre au modèle républicain, permet à la fois aux descendants d'immigrés d'accéder à l'instruction (et à la langue du pays) de manière relativement plus homogène et a priori indépendamment de leur milieu d'origine, et, in fine, d'élargir leurs possibilités d'orientation scolaire. Aux États-Unis, à l'inverse, l'école maternelle est payante et l'enseignement supérieur nettement plus coûteux qu'en France (OCDE, 2014). On pourrait ainsi supposer des écarts d'intégration dans l'emploi et de qualité de l'emploi plus réduits en France qu'aux États-Unis. Toutefois, de nombreuses études ont montré la capacité limitée de l'institution scolaire à diminuer les inégalités liées aux niveaux de capital transmis par les parents (Alba, Holdaway, 2014); la sélection migratoire initiale semble donc jouer un rôle majeur dans les inégalités entre natifs et descendants d'immigrés.

Par ailleurs, les États-Unis ont déployé depuis les années 1970 une politique de discrimination positive (affirmative action) en réponse aux discriminations observées (MaguaIn, 2006), qui a pu contribuer à améliorer l'intégration de certains descendants d'immigrés (voir tableau 8). Différentes mesures ont été mises en place dans l'éducation (telles que les quotas en fonction de l'origine dans les universités) ou sur le marché du travail qui facilitent la diminution des inégalités entre natifs et descendants d'immigrés (Holzer, Neumark, 2006). En France, l'approche républicaine de citoyenneté et d'égalité devant la loi 
rend difficile ce type de mesure, même si certains dispositifs récents de discrimination positive ont été mis en œuvre, à titre expérimental et suivant des critères de sélection d'ordre socio-économique ou de genre (comme les $\mathrm{CV}$ anonymes par exemple, BeHAGHEL et al., 2011 ou encore les quotas de femmes dans les conseils d'administration ${ }^{(14)}$ ).

En France, l'association d'institutions du marché du travail duales à des politiques ciblées d'intégration moins développées suggère une qualité de l'emploi plus élevée en moyenne qu'aux États-Unis, mais des inégalités liées à l'origine plus importantes (GALLIE, 2007). À l'inverse, les institutions du marché du travail libérales aux États-Unis mèneraient $a$ priori à des niveaux de qualité de l'emploi plus faibles mais ne favoriseraient pas les inégalités liées à l'origine. En outre, les mesures plus volontaristes d'intégration des populations immigrées et de leurs descendants concourent à réduire les inégalités liées à l'origine. $\mathrm{Au}$ niveau agrégé, nos résultats traduisent effectivement des inégalités entre natifs et descendants d'immigrés plus marquées en France qu'aux États-Unis.

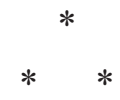

L'objectif de notre article était double. Il s'agissait, d'une part, de comparer les modèles institutionnels de la France et des États-Unis en nous intéressant à une population spécifique - les descendants d'immigrés - rarement étudiée sous cet angle; d'autre part, de prendre en compte plusieurs critères de qualité de l'emploi afin de contribuer à la littérature sur l'intégration des descendants d'immigrés dans l'emploi.

L'article a ainsi d'abord mis en lumière les fortes inégalités dans l'emploi entre natifs et descendants d'immigrés dans les deux pays. L'analyse économétrique a ensuite montré l'existence de plus fortes inégalités liées à l'origine en France qu'aux ÉtatsUnis sur l'accès à l'emploi, et une prédominance de ces inégalités liées à l'origine sur les dimensions salariale et de sécurité de l'emploi plus que sur celle du temps de travail. La désagrégation des origines a enfin mis en évidence une plus forte hétérogénéité selon l'origine en France par rapport aux États-Unis, où cette hétérogénéité reste néanmoins marquée.

Une lecture institutionnelle de nos résultats donne des éléments d'explication à ces niveaux et écarts de qualité de l'emploi. En effet, les institutions duales du marché du travail françaises semblent contribuer à accroître les inégalités entre groupes sociaux - ici les descendants d'immigrés - tout en garantissant dans l'ensemble des niveaux de qualité de l'emploi supérieurs à ceux assurés par les institutions du marché du travail américaines. Les institutions liées à l'intégration semblent jouer dans le même sens.

\section{Bibliographie}

Abowd J. M., Kramarz F., Margolis D. N., Philippon T. (2000), «The tail of two countries: minimum wages and employment in France and the United States », IZA Discussion paper, $\mathrm{n}^{\circ} 203$.

Aeberhardt R., Pouget J. (2007), «National origin wage differentials in France: evidence from matched employeremployee data», IZA Discussion paper, $\mathrm{n}^{\circ} 2779$.

Aeberhardt R., Fougère D., Pouget J., Rathelot R. (2010), «L'emploi et les salaires des enfants d'immigrés», Économie et statistique, $\mathrm{n}^{\circ}$ 433-434, pp. 31-46.

Alba R., Holdaway J. (2013), The children of immigrants at school: a comparative look at integration in the United States and Western Europe, New York, New York University Press.

(14) Voir la présentation de la «loi du 27 janvier 2001 relative à la représentation équilibrée des hommes et des femmes au sein des conseils d'administration et de surveillance et à l'égalité professionnelle»: http://www.vie-publique.fr/ actualite/panorama/texte-vote/loi-relative-representationequilibree-femmes-hommes-au-sein-conseils-administration-surveillance-egalite-professionnelle.html, consultée le 28 juillet 2015.
Algan Y., Bisin A., Verdier T. (2012), Cultural integration of immigrants in Europe, Oxford, Oxford University Press.

Amable B. (2005), Les cinq capitalismes: diversité des systèmes économiques et sociaux dans la mondialisation, Paris, Le Seuil.

Appelbaum E., Schmitt J. (2009), «Low-wage work in high-income countries: labor-market institutions and business strategy in the US and Europe», Human Relations, vol. 62, $\mathrm{n}^{\circ} 12$, pp. 1907-1934.

Behaghel L., Crépon B., Le Barbanchon T. (2011), Évaluation de l'impact du CV Anonyme, Rapport Crest/JPAL Europe/PSE.

Bernhardt A., Spiller M. W., Polson D. (2013), «All work and no pay: violations of employment and labor laws in Chicago, Los Angeles and New York City", Social forces, vol. 91, n 3 , pp. 725-746. 
BorJas G. J. (1995), «Ethnicity, neighborhoods, and human-capital externalities», The American economic review, vol. 85, n 3, pp. 365-390.

BreEM Y. (2010), «Les descendants d'immigrés», Infos migrations, $\mathrm{n}^{\circ} 15$, Paris, Ministère de 1'Immigration, de l'Intégration, de l'Identité nationale et du Développement solidaire.

Buchinsky M., Fougère D., Kramarz F., Tchernis R. (2010), «Interfirm mobility, wages and the returns to seniority and experience in the United States», The Review of economic studies, vol. 77, $\mathrm{n}^{\circ} 3$, pp. 972-1001.

Butcher K. F., DiNArdo J. (1998), «The immigrant and native born wage distributions: evidence from UnitedStates censuses », NBER working paper, $\mathrm{n}^{\circ} 6630$.

CAHuc P. (2001), «Pourquoi y a-t-il des différences de salaires ?», Reflets et perspectives de la vie économique, tome XL, $\mathrm{n}^{\circ} 1$, pp. 13-24.

Cahuc P., Kramarz F. (2004), De la précarité à la mobilité: vers une Sécurité sociale professionnelle, Rapport au ministre de l'Économie, des Finances et de l'Industrie et au ministre de l'Emploi, du Travail et de la Cohésion sociale, Paris, La Documentation française.

Cahuc P., Zajdela H. (1991), «Comment expliquer le dualisme du marché du travail à partir de comportements rationnels ?», Revue économique, vol. 42, $\mathrm{n}^{\circ} 3$, pp. 469-492.

Caroli È., Gautié J. (dir.) (2009), Bas salaires et qualité de l'emploi : l'exception française ?, Paris, Éd. Rue d'Ulm.

Davoine L., Erhel C. (2007), «La qualité de l'emploi en Europe : une approche comparative et dynamique», Économie et statistique, ${ }^{\circ} 410$, pp. 47-69.

Doomernik J., Koslowski R., Laurence J., Maxwell R., Michalowski I., ThräNhARDT D. (2009), $N^{\circ}$ shortcuts: selective migration and integration. A report to the Transatlantic academy, Washington, D. C., Transatlantic academy.

Fitoussi J.-P., Laurent É., Maurice J. (2004), «Ségrégation urbaine et intégration sociale», Rapport $d u$ Conseil d'analyse économique, $\mathrm{n}^{\circ} 45$, Paris, La Documentation française.

Gallie D. (ed.) (2007), Employment regimes and the quality of work, Oxford, Oxford University Press.

Gautié J., Schmitt J. (2010), Low-wage work in the wealthy world, New York, Russell Sage Foundation.

Glazer N., Moynihan D. P. (1963), Beyond the melting pot. The Negroes, Puerto Ricans, Jews, Italians, and Irish of New York City, Cambridge, Mass., MIT Press.

Green N. L. (1991) «L'immigration en France et aux États-Unis. Historiographie comparée», Vingtième siècle. Revue d'histoire, vol. 29, n² 29, pp. 67-82.

Guergoat-Larivière M., Marchand O. (2012), «Définition et mesure de la qualité de l'emploi : une illustration au prisme des comparaisons européennes », Économie et statistique, ${ }^{\circ} 454$, pp. 23-42.

Hasmath R. (2012), The ethnic penalty: immigration, education and the labour market, Farnham (Surrey, Royaume-Uni), Burlington (VT), Ashgate.

Heath A., Ridge J. (1983), «Social mobility of ethnic minorities», Journal of biosocial science, vol. 15, supplément S8, pp. 169-184.

Heckman J. (1998), «Detecting discrimination», Journal of economic perspectives, vol. 12, $\mathrm{n}^{\circ} 2$, pp. 101-116.

Holzer H. J., Neumark D. (2006), «Affirmative action: what do we know?», Journal of policy analysis and management, vol. 25, $\mathrm{n}^{\circ}$ 2, pp. 463-490.

Hyslop D. R. (1999), «State dependence, serial correlation and heterogeneity in intertemporal labor force participation of married women », Econometrica, vol. $67, n^{\circ} 6$, pp. 1255-1294.

INTERNATIONAL LABOUR OFFICE (ILO), INTERNATIONAL InSTITUTE FOR LABOUR STUDIES (IILS) (2008), World of work report 2008: income inequalities in the age of financial globalization, Genève, ILO.

ILO (2012), Decent work indicators: concepts and definitions, ILO Manual, première édition, Genève, ILO.

Kalleberg A. L., Reskin B. F., Hudson K. (2000), «Bad jobs in America: standard and nonstandard employment relations and job quality in the United States ", American sociological review, vol. $65, \mathrm{n}^{\circ} 2$, pp. 256-278.

LAINÉ F., OKBA M. (2005), «L'insertion des jeunes issus de l'immigration: de l'école au métier», Net. Doc., $\mathrm{n}^{\circ} 15$, Marseille, Céreq.

Le Rhun B., Minni C. (2012), «Évolution récente de l'insertion des jeunes sur le marché du travail selon le niveau de diplôme», Dares analyses, $\mathrm{n}^{\circ} 13$.

Le Rhun B., Pollet P. (2011), «Diplômes et insertion professionnelle», in Insee, France, portrait social, Paris, Insee, pp. 41-50.

Maguain D. (2006), «Discrimination positive : un bilan des expériences américaines et européennes», Revue française d'économie, vol. 21, n 2, pp. 147-193.

Meurs D., Pailhé A., Simon P. (2006), «Persistance des inégalités entre générations liées à l'immigration : l'accès à l'emploi des immigrés et de leurs descendants en France», Population, vol. 61, n 5, pp. 763-801.

Minni C., Oква M. (2014), «Emploi et chômage des descendants d'immigrés en 2012», Dares analyses, $\mathrm{n}^{\circ} 23$.

Mroz T. A. (1987), «The sensitivity of an empirical model of married women's hours to economic and statistical assumptions », Econometrica, vol. 55, $\mathrm{n}^{\circ} 4$, pp. 765-799.

ORganisation DE COOPÉRATION ET DE DÉVELOPPEMENT ÉCONOMIQUES (OCDE) (2010), Equal opportunities? The labour market integration of the children of immigrants, Paris, OCDE. 
OCDE (2012), Trouver ses marques. Les indicateurs de l'OCDE sur l'intégration des immigrés, Paris, OCDE.

OCDE (2013a), Perspectives de l'emploi de l'OCDE 2013, Paris, OCDE.

OCDE (2013b), How's Life? 2013. Measuring wellbeing, Paris, OCDE.

OCDE (2014), Regards sur l'éducation 2014: les indicateurs de l'OCDE, Paris, OCDE.

OSTERMAN P. (2013), «Introduction to the special issue on job quality: what does it mean and how might we think about it?», Industrial and labor relations review, vol. 66, $\mathrm{n}^{\circ} 4$, pp. 739-752.

Osterman P., Schulman B. (2011), Good jobs America: making work better for everyone, New York, Russell Sage Foundation.

Piketty T., Saez E. (2003), «Income inequality in the United States, 1913-1998», Quarterly journal of economics, vol. 118, $\mathrm{n}^{\circ} 1$, pp. 1-41.

Portes A., Rumbaut R. G. (2006), Immigrant America : a portrait, $3^{\text {rd }}$ ed., Berkeley, University of California Press.

Rathelot R. (2010), «Origine et quartier. Expliquer le salaire et l'emploi des descendants d'immigrés», Revue d'économie régionale \& urbaine, $\mathrm{n}^{\circ} 1, \mathrm{pp} .27-55$.
Schmitт J. (2012), Low-wage lessons, Washington D. C., Center for economic and policy research (CEPR).

Schmitt J., Jones J. (2012), Where have all the good jobs gone?, Washington D. C., Center for economic and policy research (CEPR).

Silberman R., Fournier I. (2006), «Les secondes générations sur le marché du travail en France: une pénalité ethnique ancrée dans le temps. Contribution à la théorie de l'assimilation segmentée », Revue française de sociologie, vol. 47, $\mathrm{n}^{\circ}$ 2, pp. 243-292.

Ulrich V., Zilberman S. (2007), «Six figures de l'emploi à temps partiel», Premières informations, Premières synthèses, $\mathrm{n}^{\circ}$ 39.4., Paris, Dares.

United Nations ECONOMic Commission for Europe (UNECE) (2010), Measuring quality of employment. Country pilot reports, Genève, United Nations.

WeIl P. (2003), «Races at the gate. Racial distinctions in immigration policy: a comparison between France and the United States », in Farhmeir A., Faron O., Weil P. (eds.), Migration control in the North Atlantic World: the evolution of state practices in Europe and the United States from the French Revolution to the inter-war period, New York, Berghahn Books, pp. 368-402. 


\section{Annexes}

Tableau A1 : Estimations des déterminants de la qualité de l'emploi selon l'origine en France avec une correction de l'endogénéité (avec variables de contrôle)

\begin{tabular}{|c|c|c|c|c|c|c|}
\hline \multirow[b]{2}{*}{ Variable } & \multicolumn{2}{|c|}{ Modèle 1} & \multicolumn{2}{|c|}{ Modèle 2} & \multicolumn{2}{|c|}{ Modèle 3} \\
\hline & Salaire & $\begin{array}{c}\text { Entrée dans } \\
\text { l'emploi }\end{array}$ & $\begin{array}{c}\text { Temps de } \\
\text { travail }\end{array}$ & $\begin{array}{c}\text { Entrée dans } \\
\text { l'emploi }\end{array}$ & $\begin{array}{l}\text { Sécurité de } \\
\text { l'emploi }\end{array}$ & $\begin{array}{c}\text { Entrée dans } \\
\text { l'emploi }\end{array}$ \\
\hline Non-issu de l'immigration & Ref. & Ref. & Ref. & Ref. & Ref. & Ref. \\
\hline Maghreb & $\begin{array}{r}0,034 * * * \\
(-0,010)\end{array}$ & $\begin{array}{r}-0,32 * * * \\
(-0,02)\end{array}$ & $\begin{array}{r}-0,23 * * * \\
(-0,03)\end{array}$ & $\begin{array}{r}-0,37 * * * \\
(-0,02)\end{array}$ & $\begin{array}{r}-0,14 * * * \\
(-0,03)\end{array}$ & $\begin{array}{r}-0,35 * * * \\
(-0,02)\end{array}$ \\
\hline Europe du Sud & $\begin{array}{r}-0,028 * * * \\
(-0,009)\end{array}$ & $\begin{array}{r}0,01 \\
(-0,02)\end{array}$ & $\begin{array}{r}0,04 \\
(-0,03)\end{array}$ & $\begin{array}{r}-0,01 \\
(-0,02)\end{array}$ & $\begin{array}{r}-0,01 \\
(-0,03)\end{array}$ & $\begin{array}{r}0,01 \\
(-0,02)\end{array}$ \\
\hline Reste de l'Afrique & $\begin{array}{r}-0,023 \\
(-0,021)\end{array}$ & $\begin{array}{r}-0,33 * * * \\
(-0,04)\end{array}$ & $\begin{array}{r}-0,33 * * * \\
(-0,05)\end{array}$ & $\begin{array}{r}-0,41 * * * \\
(-0,04)\end{array}$ & $\begin{array}{r}-0,05 \\
(-0,07)\end{array}$ & $\begin{array}{r}-0,38 * * * \\
(-0,04)\end{array}$ \\
\hline Europe de l'Est & $\begin{array}{r}0,010 \\
(-0,019)\end{array}$ & $\begin{array}{r}-0,14 * * * \\
(-0,04)\end{array}$ & $\begin{array}{l}-0,10^{*} \\
(-0,05)\end{array}$ & $\begin{array}{r}-0,18^{* * * *} \\
(-0,05)\end{array}$ & $\begin{array}{r}-0,11 \\
(-0,07)\end{array}$ & $\begin{array}{r}-0,18^{* * * *} \\
(-0,05)\end{array}$ \\
\hline Europe du Nord & $\begin{array}{r}0,006 \\
(-0,018)\end{array}$ & $\begin{array}{r}-0,13^{* * * *} \\
(-0,04)\end{array}$ & $\begin{array}{r}-0,11^{* *} \\
(-0,05)\end{array}$ & $\begin{array}{r}-0,15^{* * * *} \\
(-0,04)\end{array}$ & $\begin{array}{l}-0,11^{*} \\
(-0,06)\end{array}$ & $\begin{array}{r}-0,16^{* * * *} \\
(-0,04)\end{array}$ \\
\hline Etre une femme & $\begin{array}{r}-0,221 * * * \\
(-0,003)\end{array}$ & $\begin{array}{r}-0,21 * * * \\
(-0,01)\end{array}$ & $\begin{array}{r}-0,82 * * * \\
(-0,02)\end{array}$ & $\begin{array}{r}-0,30 * * * \\
(-0,01)\end{array}$ & $\begin{array}{r}-0,09 * * * \\
(-0,01)\end{array}$ & $\begin{array}{r}-0,29 * * * \\
(-0,01)\end{array}$ \\
\hline Âge & $\begin{array}{r}0,047 * * * \\
(-0,001)\end{array}$ & $\begin{array}{r}0,01 * * * \\
(0,00)\end{array}$ & $\begin{array}{r}0,04 * * * \\
(0,00)\end{array}$ & $\begin{array}{r}0,01 * * * \\
(0,00)\end{array}$ & $\begin{array}{r}0,19 * * * \\
(0,00)\end{array}$ & $\begin{array}{r}0,01 * * * \\
(0,00)\end{array}$ \\
\hline Sans diplôme & $\begin{array}{r}-0,211 * * * \\
(-0,005)\end{array}$ & $\begin{array}{r}-0,40 * * * \\
(-0,01)\end{array}$ & $\begin{array}{r}-0,38 * * * \\
(-0,01)\end{array}$ & $\begin{array}{r}-0,45 * * * \\
(-0,01)\end{array}$ & $\begin{array}{r}-0,20 * * * \\
(-0,02)\end{array}$ & $\begin{array}{r}-0,45 * * * \\
(-0,01)\end{array}$ \\
\hline Brevet des collèges/BEPC & $\begin{array}{r}-0,096^{* * *} \\
(-0,006)\end{array}$ & $\begin{array}{r}-0,14 * * * \\
(-0,01)\end{array}$ & $\begin{array}{r}-0,14 * * * \\
(-0,02)\end{array}$ & $\begin{array}{r}-0,16^{* * * *} \\
(-0,01)\end{array}$ & $\begin{array}{r}-0,10 * * * \\
(-0,02)\end{array}$ & $\begin{array}{r}-0,15^{* * * *} \\
(-0,01)\end{array}$ \\
\hline $\mathrm{BEP} / \mathrm{CAP}$ & $\begin{array}{r}-0,156^{* * *} \\
(-0,004)\end{array}$ & $\begin{array}{r}0,03 * * * \\
(-0,01)\end{array}$ & $\begin{array}{r}0,01 \\
(-0,01)\end{array}$ & $\begin{array}{r}0,04 * * * \\
(-0,01)\end{array}$ & $\begin{array}{r}-0,08 * * * \\
(-0,02)\end{array}$ & $\begin{array}{r}0,04 * * * \\
(-0,01)\end{array}$ \\
\hline Baccalauréat & Ref. & Ref. & Ref. & Ref. & Ref. & Ref. \\
\hline $\mathrm{Bac}+2$ & $\begin{array}{r}0,093 * * * \\
(-0,005)\end{array}$ & $\begin{array}{r}0,30 * * * \\
(-0,01)\end{array}$ & $\begin{array}{r}0,26 * * * \\
(-0,01)\end{array}$ & $\begin{array}{r}0,36 * * * \\
(-0,01)\end{array}$ & $\begin{array}{r}0,09 * * * \\
(-0,02)\end{array}$ & $\begin{array}{r}0,37 * * * \\
(-0,01)\end{array}$ \\
\hline Licence ou plus & $\begin{array}{r}0,294 * * * \\
(-0,005)\end{array}$ & $\begin{array}{r}0,33 * * * \\
(-0,01)\end{array}$ & $\begin{array}{r}0,28 * * * \\
(-0,01)\end{array}$ & $\begin{array}{r}0,33 * * * \\
(-0,01)\end{array}$ & $\begin{array}{r}0,10^{* * *} \\
(-0,02)\end{array}$ & $\begin{array}{r}0,33 * * * \\
(-0,01)\end{array}$ \\
\hline Habiter en province hors d'une ZUS & Ref. & Ref. & Ref. & Ref. & Ref. & Ref. \\
\hline Habiter en région parisienne hors d'une ZUS & $\begin{array}{r}0,168 * * * \\
(-0,004)\end{array}$ & $\begin{array}{r}0,09 * * * \\
(-0,01)\end{array}$ & $\begin{array}{r}0,24 * * * \\
(-0,01)\end{array}$ & $\begin{array}{r}0,09 * * * \\
(-0,01)\end{array}$ & $\begin{array}{r}0,15 * * * \\
(-0,02)\end{array}$ & $\begin{array}{r}0,10 * * * \\
(-0,01)\end{array}$ \\
\hline Habiter en province dans une ZUS & $\begin{array}{r}0,004 \\
(-0,009)\end{array}$ & $\begin{array}{r}-0,22 * * * \\
(-0,02)\end{array}$ & $\begin{array}{r}-0,17 * * * \\
(-0,02)\end{array}$ & $\begin{array}{r}-0,24 * * * \\
(-0,02)\end{array}$ & $\begin{array}{r}-0,08 * * * \\
(-0,03)\end{array}$ & $\begin{array}{r}-0,23 * * * \\
(-0,02)\end{array}$ \\
\hline Habiter en région parisienne dans une ZUS & $\begin{array}{r}0,074 * * * \\
(-0,016)\end{array}$ & $\begin{array}{r}-0,03 \\
(-0,04)\end{array}$ & $\begin{array}{r}0,21 * * * \\
(-0,04)\end{array}$ & $\begin{array}{r}-0,01 \\
(-0,04)\end{array}$ & $\begin{array}{r}0,09 \\
(-0,06)\end{array}$ & $\begin{array}{r}0,01 \\
(-0,04)\end{array}$ \\
\hline Avoir un seul parent immigré & $\begin{array}{l}0,027 * * \\
(-0,011)\end{array}$ & $\begin{array}{r}-0,03 \\
(-0,02)\end{array}$ & $\begin{array}{r}-0,04 \\
(-0,03)\end{array}$ & $\begin{array}{r}-0,03 \\
(-0,02)\end{array}$ & $\begin{array}{r}0,02 \\
(-0,04)\end{array}$ & $\begin{array}{r}-0,04 \\
(-0,02)\end{array}$ \\
\hline Ne pas avoir d'enfant & Ref. & Ref. & Ref. & Ref. & Ref. & Ref. \\
\hline Avoir un enfant & & $\begin{array}{r}0,04 * * * \\
(-0,01)\end{array}$ & & $\begin{array}{r}0,10 * * * \\
(-0,01)\end{array}$ & & $\begin{array}{r}0,08 * * * \\
(-0,01)\end{array}$ \\
\hline Deux enfants & & $\begin{array}{r}0,09 * * * \\
(-0,01)\end{array}$ & & $\begin{array}{r}0,23 * * * \\
(-0,01)\end{array}$ & & $\begin{array}{r}0,16^{* * *} \\
(-0,01)\end{array}$ \\
\hline Trois enfants & & $\begin{array}{r}-0,14 * * * \\
(-0,01)\end{array}$ & & $\begin{array}{r}0,03 * * * \\
(-0,01)\end{array}$ & & $\begin{array}{r}-0,16^{* * * *} \\
(-0,01)\end{array}$ \\
\hline Être en couple & & $\begin{array}{r}0,18^{* * *} \\
(-0,01)\end{array}$ & & $\begin{array}{r}0,18 * * * \\
(-0,01)\end{array}$ & & $\begin{array}{r}0,23 * * * \\
(-0,01)\end{array}$ \\
\hline Constante & $\begin{array}{r}6,434 * * * \\
(-0,019)\end{array}$ & $\begin{array}{r}0,44 * * * \\
(-0,02)\end{array}$ & $\begin{array}{l}-0,11^{*} \\
(-0,06)\end{array}$ & $\begin{array}{r}0,39 * * * \\
(-0,02)\end{array}$ & $\begin{array}{r}-2,51 * * * \\
(-0,08)\end{array}$ & $\begin{array}{r}0,46 * * * \\
(-0,02)\end{array}$ \\
\hline $\begin{array}{l}\text { Rho } \\
\text { Observations }\end{array}$ & $\begin{array}{r}-0,845^{* * * *} \\
144591\end{array}$ & 152472 & $\begin{array}{r}0,864 * * * \\
144591\end{array}$ & 152472 & $\begin{array}{r}-0,443 * * * \\
144591\end{array}$ & 152472 \\
\hline
\end{tabular}

Variables contrôlées disponibles sur demande : âge², secteur d'activité, année.

Notes : La régression sur le salaire est linéaire (MCO), les régressions sur le temps de travail et sur la sécurité de l'emploi sont des probits binomiaux. Les symboles *, ${ }^{*}$ et $* * *$ représentent les seuils de significativité statistique à respectivement 10,5 et $1 \%$.

Champ : Natifs et descendants d'immigrés en emploi, de 20 à 60 ans, en France métropolitaine.

Source : Enquêtes Emploi en continu 2008-2012, Insee. 
Tableau A2 : Estimations des déterminants de la qualité de l'emploi selon l'origine aux États-Unis avec une correction de l'endogénéité (avec variables de contrôle)

\begin{tabular}{|c|c|c|c|c|c|c|}
\hline \multirow[b]{2}{*}{ Variable } & \multicolumn{2}{|c|}{ Modèle 1} & \multicolumn{2}{|c|}{ Modèle 2} & \multicolumn{2}{|c|}{ Modèle 3} \\
\hline & Salaire & $\begin{array}{c}\text { Entrée dans } \\
\text { l'emploi }\end{array}$ & $\begin{array}{l}\text { Temps de } \\
\text { travail }\end{array}$ & $\begin{array}{l}\text { Entrée dans } \\
\text { l'emploi }\end{array}$ & $\begin{array}{c}\text { Sécurité de } \\
\text { l'emploi }\end{array}$ & $\begin{array}{c}\text { Entrée dans } \\
\text { l'emploi }\end{array}$ \\
\hline Non-issu de l'immigration & Ref. & Ref. & Ref. & Ref. & Ref. & Ref. \\
\hline \multirow[t]{2}{*}{ Mexique } & $-0,010$ & 0,00 & 0,03 & 0,03 & $-0,01$ & 0,04 \\
\hline & $(-0,014)$ & $(-0,03)$ & $(-0,03)$ & $(-0,03)$ & $(-0,02)$ & $(-0,03)$ \\
\hline \multirow[t]{2}{*}{ Porto Rico } & $-0,020$ & 0,01 & 0,01 & 0,07 & $0,06^{*}$ & 0,07 \\
\hline & $(-0,024)$ & $(-0,05)$ & $(-0,06)$ & $(-0,05)$ & $(-0,04)$ & $(-0,05)$ \\
\hline \multirow[t]{2}{*}{ Asie } & $0,144 * * *$ & 0,05 & $-0,03$ & 0,04 & $0,06^{*}$ & 0,04 \\
\hline & $(-0,023)$ & $(-0,05)$ & $(-0,06)$ & $(-0,05)$ & $(-0,04)$ & $(-0,05)$ \\
\hline \multirow[t]{2}{*}{ Canada } & $0,065^{* *}$ & 0,06 & $-0,05$ & 0,08 & $-0,02$ & 0,05 \\
\hline & $(-0,025)$ & $(-0,05)$ & $(-0,06)$ & $(-0,06)$ & $(-0,04)$ & $(-0,06)$ \\
\hline \multirow[t]{2}{*}{ Europe du Nord } & $0,078 * * *$ & 0,03 & 0,01 & 0,01 & $-0,05$ & 0,01 \\
\hline & $(-0,020)$ & $(-0,04)$ & $(-0,05)$ & $(-0,05)$ & $(-0,03)$ & $(-0,05)$ \\
\hline \multirow[t]{2}{*}{ Europe du Sud } & $0,129 * * *$ & 0,04 & $-0,08$ & 0,00 & 0,03 & $-0,01$ \\
\hline & $(-0,021)$ & $(-0,04)$ & $(-0,05)$ & $(-0,05)$ & $(-0,03)$ & $(-0,05)$ \\
\hline \multirow[t]{2}{*}{ Europe de l'Est } & $0,098 * * *$ & 0,13 & $-0,01$ & $0,22 * *$ & $-0,07$ & $0,22 * *$ \\
\hline & $(-0,033)$ & $(-0,08)$ & $(-0,08)$ & $(-0,09)$ & $(-0,05)$ & $(-0,09)$ \\
\hline \multirow[t]{2}{*}{ Caraïbes } & 0,071 & $-0,25 * * *$ & $0,28 * *$ & $-0,28 * * *$ & $-0,08$ & $-0,25 * * *$ \\
\hline & $(-0,044)$ & $(-0,07)$ & $(-0,12)$ & $(-0,08)$ & $(-0,06)$ & $(-0,08)$ \\
\hline \multirow[t]{2}{*}{ Amérique latine } & 0,015 & $-0,01$ & 0,02 & 0,00 & $-0,07$ & 0,00 \\
\hline & $(-0,032)$ & $(-0,06)$ & $(-0,08)$ & $(-0,07)$ & $(-0,05)$ & $(-0,07)$ \\
\hline \multirow[t]{2}{*}{ Autres } & $0,078 * * *$ & 0,04 & $-0,11^{*}$ & 0,05 & $-0,08 * *$ & 0,06 \\
\hline & $(-0,026)$ & $(-0,05)$ & $(-0,06)$ & $(-0,06)$ & $(-0,04)$ & $(-0,06)$ \\
\hline \multirow[t]{2}{*}{ Être une femme } & $-0,482 * * *$ & $-0,0503 * * *$ & $-0,45^{* * *}$ & $-0,10^{* * *}$ & $-0,40 * * *$ & $-0,12 * * *$ \\
\hline & $(-0,004)$ & $(-0,01)$ & $(-0,01)$ & $(-0,01)$ & $(-0,01)$ & $(-0,01)$ \\
\hline \multirow[t]{2}{*}{ Âge } & $0,067 * * *$ & $0,01 * * *$ & $0,04 * * *$ & $0,01 * * *$ & $0,02 * * *$ & $0,01 * * *$ \\
\hline & $(-0,001)$ & $(0,00)$ & $(0,00)$ & $(0,00)$ & $(0,00)$ & $(0,00)$ \\
\hline \multirow[t]{2}{*}{ Sans diplôme } & $-0,385 * * *$ & $-0,17 * * *$ & $-0,36 * * *$ & $-0,30 * * *$ & $-0,52 * * *$ & $-0,30 * * *$ \\
\hline & $(-0,025)$ & $(-0,04)$ & $(-0,05)$ & $(-0,04)$ & $(-0,04)$ & $(-0,04)$ \\
\hline \multirow[t]{2}{*}{ Sortie du lycée sans diplôme } & $-0,228 * * *$ & $-0,28 * * *$ & $-0,20 * * *$ & $-0,37 * * *$ & $-0,41 * * *$ & $-0,36^{* * *}$ \\
\hline & $(-0,010)$ & $(-0,02)$ & $(-0,02)$ & $(-0,02)$ & $(-0,01)$ & $(-0,02)$ \\
\hline Diplôme du lycée & Ref. & Ref. & Ref. & Ref. & Ref. & Ref. \\
\hline \multirow{2}{*}{$\mathrm{Bac}+2$} & $0,151 * * *$ & $0,16 * * *$ & 0,02 & $0,20 * * *$ & $0,14 * * *$ & $0,19 * * *$ \\
\hline & $(-0,006)$ & $(-0,01)$ & $(-0,01)$ & $(-0,01)$ & $(-0,01)$ & $(-0,01)$ \\
\hline Licence ou plus & $0,488 * * *$ & $0,39 * * *$ & $0,20 * * *$ & $0,37 * * *$ & $0,31 * * *$ & $0,35 * * *$ \\
\hline & $(-0,004)$ & $(-0,01)$ & $(-0,01)$ & $(-0,01)$ & $(-0,01)$ & $(-0,01)$ \\
\hline Résidence & $-0,182 * * *$ & $-0,02 *$ & $-0,07 * * *$ & $-0,01$ & $-0,05^{* * *}$ & $-0,01$ \\
\hline & $(-0,004)$ & $(-0,01)$ & $(-0,01)$ & $(-0,01)$ & $(-0,01)$ & $(-0,01)$ \\
\hline Avoir un seul parent immigré & $-0,030 * *$ & 0,00 & $-0,05$ & $-0,02$ & 0,02 & $-0,02$ \\
\hline & $(-0,015)$ & $(-0,03)$ & $(-0,03)$ & $(-0,03)$ & $(-0,02)$ & $(-0,03)$ \\
\hline Ne pas avoir d'enfant & & Ref. & & Ref. & & Ref. \\
\hline Avoir un enfant & & $0,08 * * *$ & & $0,07 * * *$ & & $0,08 * * *$ \\
\hline & & $(-0,01)$ & & $(-0,01)$ & & $(-0,01)$ \\
\hline Deux enfants & & $0,13 * * *$ & & $0,11 * * *$ & & $0,14 * * *$ \\
\hline & & $(-0,01)$ & & $(-0,01)$ & & $(-0,01)$ \\
\hline Trois enfants & & $0,06^{* * *}$ & & 0,00 & & $0,06^{* * *}$ \\
\hline & & $(-0,01)$ & & $(-0,01)$ & & $(-0,01)$ \\
\hline Être en couple & & $0,19 * * *$ & & $0,20 * * *$ & & $0,27 * * *$ \\
\hline & & $(-0,01)$ & & $(-0,01)$ & & $(-0,01)$ \\
\hline Constante & $-13,920 * * *$ & 7,45 & $69,95 * * *$ & $14,43^{* * *}$ & $37,63 * * *$ & $9,49^{*}$ \\
\hline & $(-2,483)$ & $(-5,09)$ & $(-5,95)$ & $(-5,43)$ & $(-3,74)$ & $(-5,41)$ \\
\hline Rho & $-0,848 * * *$ & & $-0,725 * * *$ & & $0,932 * * *$ & \\
\hline Observations & 153422 & 244701 & 153422 & 244701 & 153422 & 244701 \\
\hline
\end{tabular}

Variables contrôlées disponibles sur demande : âge², secteur d'activité, année.

Notes : La régression sur le salaire est linéaire (MCO), les régressions sur le temps de travail et sur la sécurité de l'emploi sont des probits binomiaux. Les symboles *, ${ }^{* *}$ et $* * *$ représentent les seuils de significativité statistique à respectivement 10,5 et $1 \%$.

Champ : Natifs et descendants d'immigrés en emploi, de 20 à 60 ans, aux États-Unis.

Source : Current Population Surveys 2008-2012, BLS. 